\title{
The Effects of a Six Week Lumbopelvic Control and Balance Training Program in High School Basketball Players
}

Margaret Long

Follow this and additional works at: https://researchrepository.wvu.edu/etd

\section{Recommended Citation}

Long, Margaret, "The Effects of a Six Week Lumbopelvic Control and Balance Training Program in High School Basketball Players" (2017). Graduate Theses, Dissertations, and Problem Reports. 6109. https://researchrepository.wvu.edu/etd/6109

This Thesis is protected by copyright and/or related rights. It has been brought to you by the The Research Repository @ WVU with permission from the rights-holder(s). You are free to use this Thesis in any way that is permitted by the copyright and related rights legislation that applies to your use. For other uses you must obtain permission from the rights-holder(s) directly, unless additional rights are indicated by a Creative Commons license in the record and/ or on the work itself. This Thesis has been accepted for inclusion in WVU Graduate Theses, Dissertations, and Problem Reports collection by an authorized administrator of The Research Repository @ WVU. For more information, please contact researchrepository@mail.wvu.edu. 
The Effects of a Six Week Lumbopelvic Control and Balance Training Program in High School Basketball Players

Margaret Long LAT, ATC

Thesis submitted to the

College of Physical Activity and Sport Sciences

at West Virginia University

in partial fulfillment of the requirements

for the degree of

Masters of Science

in

Athletic Training

Michelle A. Sandrey, PhD, ATC, Chair

Jean McCrory, $\mathrm{PhD}$

Allison Hetrick, M. Ed, ATC, CSCS

Department of Sport Sciences

Morgantown, WV

2017

Keywords: lumbopelvic control, balance, high school basketball

Copyright 2017 Margaret Long 


\begin{abstract}
The Effects of a Six Week Lumbopelvic Control and Balance Training Program in High School Basketball Players
\end{abstract}

Margaret Long LAT, ATC

Context: Lumbopelvic control (LPC) and balance training programs are popular in the sport of basketball, but there is little research to indicate whether significant improvements in lumbopelvic control and balance are evident after completion of a training program. Objective: The objective of this study is to determine if conducting a six week lumbopelvic control and balance training program will lead to improvement in lumbopelvic control and balance in high school male basketball players. Design: This study will be a repeated measures design. Setting: High School in Western Pennsylvania. Patients and Other participants: This study includes 15 varsity and junior varsity basketball players. Fourteen players completed the study. The average age was $15.21 \pm 0.71$ years old. Average height was $180.82 \pm 1.8 \mathrm{~cm}$, and average weight was $69.63 \pm 0.64 \mathrm{~kg}$. Inclusion criteria was healthy subjects who have not had shoulder, hip, or abdominal surgery within the year and have not had a shoulder, hip, or abdominal injury in the past six months. All participants were members of the boys' basketball team at the high school with a sport physical on file. Exclusion criteria will include those who have had shoulder, hip or abdominal surgery within the past year and those who have had a shoulder, hip or abdominal injury within the past six months. Interventions: The single leg raise test (SLRT) abdominal fatigue test (AFT), and the modified star excursion balance test (SEBT) were completed before and after performing a six week lumbopelvic and balance training program two times a week. The training program consisted of a progression of lumbopelvic and balance exercises based on level of difficulty and conducive to the sport of basketball. Main Outcome Measures: The dependent variables are the distances reached for both legs in three directions (anterior, posteromedial, and posterolateral) of the SEBT, degrees of anterior/posterior tilt of the pelvis in SLRT, and time held in AFT. Results: Significant differences were shown in the SLRT left leg $\left(\mathrm{F}_{1,13}=7.556, \mathrm{P}=0.017, \mathrm{ES}=-0.80,95 \% \mathrm{CI}=-1.57\right.$ to -0.03$)$. No significant differences were shown in the SLRT right leg, AFT, or SEBT in all directions. Large effect sizes were shown in AFT, all right leg SEBT directions, and SEBT left leg anterior direction. Moderate effect sizes were shown in SLRT right leg, SEBT posterolateral left leg, and SEBT posteromedial left leg. Conclusion: The six-week LPC and balance intervention program could be used to increase LPC, but do not show an ability to improve dynamic balance 


\section{ACKNOWLEDGEMENTS}

I would like to start by thanking the Waynesburg Central High School boys' basketball team. I am very grateful for everyone's cooperation for completing the exercises and completing all the testing. I would not have completed this study if it was not for your cooperation.

Thank you to Kirk King, Head WCHS Boys' Basketball coach, for allowing me to use your team for my study and allowing me to use a portion of your practice time to implement the exercise protocols. I would also like to thank the assistant coaches who helped to instruct and supervise the exercises.

Thank you to my committee chair and graduate advisor, Dr. Michelle Sandrey. I appreciate all the hours that were put into editing my thesis and all the advice to make my study the best it could be.

Thank you to my committee members, Allison Hetrick and Dr. Jean McCrory. I am very appreciative of the time and effort you have put into this process. 
ACKNOWLEDGEMENTS

iii

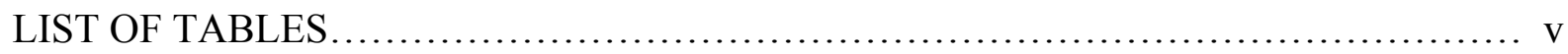

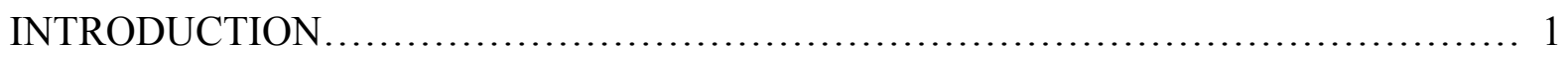

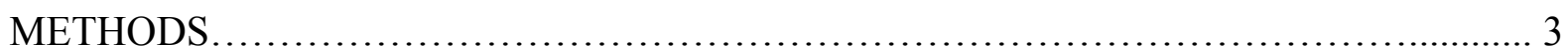

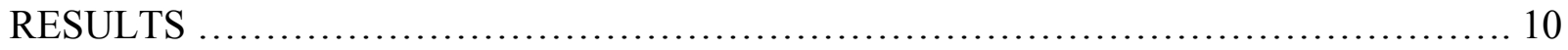

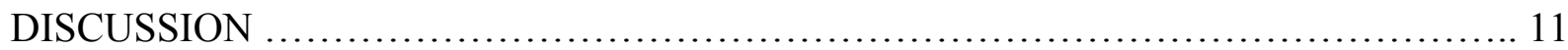

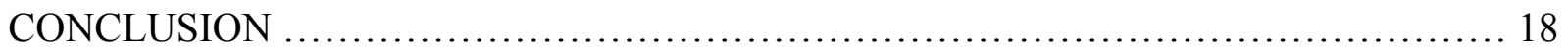

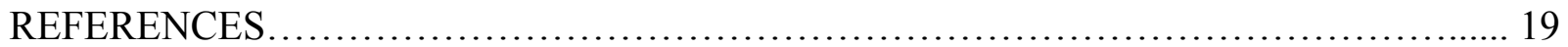

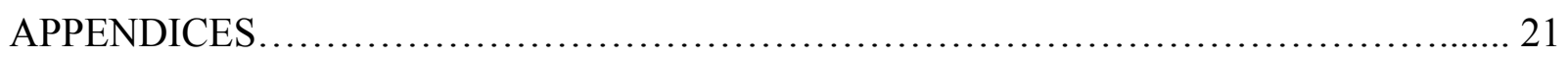

APPENDIX A: THE PROBLEM...................................................... 22

APPENDIX B: LITERATURE REVIEW ........................................ 28

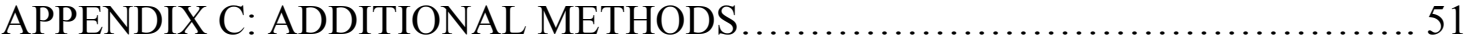

APPENDIX D: ADDITIONAL RESULTS ..................................... 86

APPENDIX E: RECOMMENDATION FOR FUTURE RESEARCH .............. 87

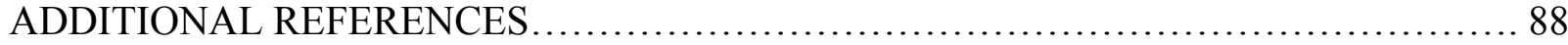


LIST OF TABLES Page

Table B1. Muscle Origin/Insertion/Action of Lumbopelvic-Hip Complex........... 32

Table B2. Other Measurements of Lumbopelvic Control............................ 38

Table B3. Intervention Exercises for Lumbopelvic Control........................ 45

Table B4. Intervention Exercises for Balance.................................. 47

Table C1. Informed Consent................................................ 51

Table C2. Informed Assent................................................ 55

Table C3. Subject Demographics............................................. 58

Table C4. Data Collection.................................................... 59

Table C5. Lumbopelvic Control/Balance Training Intervention Program.............. 61

Table C6. Pre-test/Post test................................................ 63

Table C7. Lumbopelvic Control Intervention Week 1 Exercises …................ 65

Table C8. Lumbopelvic Control Intervention Week 2 Exercises .................. 67

Table C9. Lumbopelvic Control Intervention Week 3 Exercises .................. 70

Table C10. Lumbopelvic Control Intervention Week 4 Exercises ................... 74

Table C11. Lumbopelvic Control Intervention Week 5 Exercises …............... 78

Table C12. Lumbopelvic Control Intervention Week 6 Exercises ................... 82

Table D1. Descriptive Statistics on Means and Standard Deviations for

Dependent Variables............................................ 86

Table D2. Repeated Measures ANOVA Results ................................. 86 


\section{INTRODUCTION}

Basketball is one of the most popular sports in high school. In 2013, the NCAA reported 538,676 athletes were playing basketball at the high school level. ${ }^{1}$ Along with the increased participation rate, the risk of injury increased as well. The highest injury rates are to the ankle/foot and knee. ${ }^{2}$ It has been suggested that prevention programs may help to decrease those injury rates. ${ }^{3,4}$ Plisky et al. ${ }^{3}$ stated that neuromuscular control can be increased to help prevent knee injuries. Chaudhari et al. ${ }^{4}$ concluded that poor lumbopelvic control (LPC) was correlated with higher injury rates. Thus, balance and LPC exercises should be incorporated into rehabilitation programs as increases in balance and LPC have shown decreases in injury. ${ }^{3,5}$ As most programs are based on performance enhancement and injury prevention, the issue is that there is limited research conducted on the relationship of these programs to basketball let alone how the exercises incorporated relate to balance and LPC, which are two key elements important to basketball.

An athlete must maintain an equal amount of weight surrounding the center of gravity in order to maintain balance. ${ }^{6}$ When an athlete, especially a basketball athlete, maintains a spine neutral position, the trunk is in a neutral position causing the body to equally distribute weight

over the center of gravity. ${ }^{7,8}$ During basketball skills, this is very important for accelerations and decelerations on the court, change of direction, penetrations into the defensive perimeter, boxing out, dribbling, and defensive position recovery; ${ }^{6,8}$ Bishop and $\mathrm{Hay}^{7}$ hypothesized that the center of gravity moves in relation to the athlete's trunk. A disequilibrium of the athlete could occur with many movements including twisting movement of the feet, jump shots, and offensive/defensive rebounds. ${ }^{6,9}$ A disruption in the equilibrium could cause a disruption in the athlete's performance by the unequal balance of weight over the center of gravity throwing the 
athlete off balance. ${ }^{6,10}$ Therefore, an increase in LPC is important to control and maintain the spine neutral position for the lumbar spine and pelvis. Thus, a spine neutral position is better maintained and controlled by increasing the amount of LPC the athlete has. This concept can be generalized to describe the lumbopelvic-hip complex (LPHC) remaining in a stable position in order to oppose internal and external perturbations. ${ }^{4}$

Balance results from neuromuscular actions in response to continuous visual, vestibular, and somato-sensory feedback. ${ }^{6}$ Stabilization to internal and external perturbations are necessary in order to reduce abnormal perturbations, which would cause disequilibrium. ${ }^{6}$ As balance plays a crucial role in everyday activities such as walking and running, ${ }^{8,9}$ and all sport activities, ${ }^{9}$ especially basketball, ${ }^{10}$ enhancement of balance should be considered. Further, basketball players also need to have not only an awareness of the body, but of the ball as well. ${ }^{10}$ Though there is not sound evidence to show an increase in balance can increase basketball movements; there is enough research to show that balance plays a crucial role in the sport of basketball when performing certain skills. ${ }^{11}$

Postural control and load forces placed on the body are considered, ${ }^{12}$ using the focus of the neuromuscular system, to control and protect the lumbar spine and pelvis from abnormal movements in all three planes (frontal, sagittal, and transverse) and maintain a neutral position. ${ }^{4,}$ ${ }^{12-14}$ Thus, spinal control and balance create a relationship between LPC and the neuromuscular system. Therefore, an increase in LPC is important to control and maintain the lumbar spine and pelvis to maintain the spine neutral position. As spine neutral position is better maintained and controlled by increasing LPC and balance the basketball athlete has, this should be incorporated in a training program for basketball. However, little guidance has been provided in the literature for constructing a LPC and balance training program. Suggested activities include pelvic spine 
neutral, core stabilization, single leg balance, diagonal movements and movements conducive to basketball while maintaining spine neutral and contraction of the LPHC muscles. ${ }^{15,16}$ Unfortunately, there is no research relating these results to high school basketball to the author's knowledge as there was in a soccer study. ${ }^{17}$ Thus, more research should be established on LPC and balance training for high school basketball players. The purpose of this study is to determine if conducting a six week LPC and balance training program will cause increases in LPC and balance in high school male basketball players.

\section{METHODS}

This study was a repeated measures design. The testing was time dependent in the manner of a pre-test and post-test protocol. The independent variables were Time (pre/posttest) for the Abdominal Fatigue Test (AFT), Time and Direction (anterior, posteromedial and posterolateral) for the Star Excursion Balance Test (SEBT), and Time and Degrees (anterior/ posterior tilt) for the Single Leg Raise Test (SLRT). The dependent variables were the distances reached in each direction (anterior, posteromedial, and posterolateral) of the SEBT, degrees of anterior/posterior tilt of pelvis in the SLRT, and time held in the AFT. Subjects

This study included 15 varsity and junior varsity basketball players from a high school in western Pennsylvania. Fourteen subjects completed the study in entirety. The average age of the athletes was $15.21 \pm 0.71$ years old. Average height was $180.82 \pm 1.8 \mathrm{~cm}$, and average weight was $69.63 \pm 0.64 \mathrm{~kg}$. Inclusion criteria included those subjects who are healthy, have no disorders affecting balance or neuromuscular control, no history of an injury to the shoulder in the past six months, and no history of surgeries to the core or hips within the past year. The subject had a sport physical on file and was a member of the boys' basketball team during the duration of the 
study. Exclusion criteria included subjects who have a history of surgery to the core or hips within the past year, those who have a disorder affecting balance and neuromuscular control, and those taking medication that may affect balance and neuromuscular control. To remain in the study, subjects must have completed $75 \%$ of the intervention ( 9 sessions). This study was approved by the Institutions Office of Research Compliance.

Procedures

Before the pre-testing procedures and the functional training program were implemented, an informational meeting took place with the subjects and their parents. In this meeting, the informed parental consent form with HIPPA included (Table C1), informed assent form (Table $\mathrm{C} 2$ ), and the demographic questionnaire (Table C3) were discussed. The informed parental consent with HIPPA and demographic questionnaire was completed during this informational meeting. Once subjects and parents completed the necessary paperwork, testing procedures (Table C3) as well as the functional training progression (Table C4) were explained as well. Instructions for the testing procedures were explained to all subjects during the informational meeting and before performing the tests. It was also explained that, during testing days, subjects will not participate in any personal workouts that included cardiovascular training and strength training.

The week following the informational meeting, all subjects completed pre-testing procedures in order to establish baseline values. Pre-testing procedures included the Single Leg Raise Test, Abdominal Fatigue Test, and the Star Excursion Balance Test (Table C5). Prior to all testing, procedures and instrumentation were described to the subject. One researcher performed all testing and training procedures to maintain consistency.

Following the pre-testing procedures, all experimental subjects participated in the 
lumbopelvic control/balance training intervention program (Table C6). Exercises for each phase were performed twice per week, with each session occurring at the beginning of a practice session. Sets and repetitions for each exercise can be found in Table C6. In this program, progression was based on difficulty of exercise by phases and increases in sets and reps for each phase occurred when the subject was ready to progress in order to target the integration of exercises from isolated segments to full body movement to functional sport related activities. Pre-test/Post-test (Table C5)

Pre-test procedures were completed prior to the start of the intervention training program. Post-testing procedures were conducted the week after the conclusion of the lumbopelvic control/balance program. These testing procedures were the same as the pre-testing procedures. Data was collected by the co-principal investigator (Table C4).

Single leg raise test (SLRT) ${ }^{4,18}$ : The single leg raise test was performed by placing the CoreX belt with iPod-based sensor (Level Belt Pro application) level on the hips and lined with the ASIS and PSIS. The athlete stood with feet shoulder width apart then slowly raised one leg three inches off the ground and slowly lowered it back down. The anterior/posterior pelvic tilt measurement was recorded by taking the largest of two measurements recorded on the app. The belt was taken off then immediately placed back on to realign the belt with the ASIS and the PSIS. The test was then repeated. The two scores were then averaged for the final score. The SLRT showed good intra-rater reliability. ${ }^{19}$ The SLRT was compared to 3D motion analysis for validation showing high sensitivity, specificity, and accuracy. ${ }^{4}$

Abdominal fatigue test (AFT) ${ }^{5,20-22}$ : The test was performed with the athlete seated on a table with the hips and knees at a 90-degree angle with the upper body at 60-degrees in relation to the table. The feet were strapped to the table, and the athlete held the 60-degree angle for as long as possible. Anytime the position was broken the trial was not valid. The time the athlete 
held the correct position was recorded. Test re-test reliability was found to have ICC scores of 0.95-0.98. Endurance times and ratios between tests are used as guidelines to interpret results. Star excursion balance test (SEBT) $)^{3,17,23-25}$ The athlete began in the starting position with both feet flat on the ground in the center of the grid with hands on both hips at all times. The stance foot was directly on the ground without lifting the heel while reaching with the other leg in one direction. The athlete tried to touch the tape measure as far as possible with the most distal part of the foot once. After the touch, the athlete returned to the starting position. This protocol was repeated for the other two directions with the start direction and the other two directions randomized. Once the three directions (anterior, posterolateral, posteromedial) were completed the stance leg was switched after a five-minute rest period. The athlete was allowed four practice rounds before true measurements were taken. The trial was not counted if the athlete tapped the foot on the ground more than once, slid the reach foot, missed the tape measure with the tap, pushed off the floor with the reach foot, lifted stance heel off the ground, took the hand off the hips, or was unable to return reach foot to starting stance. ${ }^{24}$ Leg length (from ASIS to medial malleolus) were measured in order to determine normalization. Each direction measurement was recorded. ICC results was 0.88 for the anterior direction, 0.91 for the posteromedial direction, and 0.88 for the posterolateral direction. ${ }^{23}$ The modified SEBT was used as the three directions are the most travelled during activity, especially basketball. ${ }^{3}$ Intervention Program (Table C6)

All the exercises in this intervention protocol were taken from previous studies and chosen for the relationship with movements of basketball. ${ }^{5,11,15,22,26-29}$ All exercises began with contraction and static hold of LPHC musculature to improve proper activation. ${ }^{15}$ The exercises progressed to more advanced contract and static hold in quadruped and standing positions. ${ }^{15}$ The 
program finally progressed to functional sport specific exercises while maintaining the activation of the LPHC. ${ }^{15}$ The exercises were performed in phases. All the subjects began on phase 1. When they successfully performed two consecutive sessions of the exercise, the subject moved to the next phase.

Intervention

Phase 1 (Table C7): During the first phase, the subject performed supine abdominal hollowing ( 3 sets $30 \mathrm{sec}$. hold), supine abdominal bracing ( 3 sets $30 \mathrm{sec}$. hold), prone bridge (3 sets 30 sec. hold), supine bridge ( 3 sets 30 sec. hold), curl up ( 3 sets, 15 reps), superman ( 3 sets $30 \mathrm{sec}$. hold), and cat/camel exercises ( $3 \mathrm{sets}, 15 \mathrm{sec}$. each position). These exercises focused on the contraction and static hold of LPHC musculature to improve activation.

Phase 2 (Table C8): During the second phase, the subject performed supine knee raises (3 sets $30 \mathrm{sec}$. hold), side bridges ( 3 sets $30 \mathrm{sec}$. hold each side), supine single leg bridges ( 3 sets 30 sec. hold), standing abdominal hollowing (3 sets 30 sec. hold), standing abdominal bracing (3 sets $30 \mathrm{sec}$. hold), aqua man ( 3 sets, 15 reps), cat/camel exercises with eyes closed ( 3 sets, 15 sec. each position). These exercises were a continuation of phase 1 with added difficulty by adding a gravity resisted position while still maintaining activation of the LPHC during exercises.

Phase 3 (Table C9): During the third phase, the subject performed prone plank (3 sets 30 sec. hold), bird dog ( 3 sets, 15 reps), glut side lying snaps ( 3 sets, 15 reps each side), bridges on physioball ( 3 sets 30 sec. hold), push through ( 3 sets, 15 reps), cross over crunch ( 3 sets, 15 reps), single leg lift ( 3 sets, 15 reps), and medicine ball sit up (3 sets, 15 reps). This phase began incorporating quadruped and standing positions while maintaining activation of LPHC. 
Phase 4 (Table C10): During the fourth phase, the subject performed side plank (3 sets 30 sec. hold), dead bug ( 3 sets, 15 reps), bicycles ( 3 sets, 15 reps), march ups on physioball ( 3 sets, 15 reps each side), apollo creed ( 3 sets, 15 reps), medicine ball floor twist ( 3 sets, 15 reps), double leg lift (3 sets, 15 reps), and swimming on knees ( 3 sets, 15 reps). This phase repeated phase 3 by continuing the quadruped and standing position while maintaining activation of LPHC, but now the positions were working against gravity adding difficulty.

Phase 5 (Table C11): During the fifth phase, the subject performed a forward lunge with medicine ball twist ( 3 sets, 15 reps), standing reverse wood chop ( 3 sets, 15 reps each side), single leg squat ( 3 sets, 15 reps each side), bosu squats ( 3 sets, 15 reps), push-ups ( 3 sets, 15 reps), medicine ball throw ( 3 sets, 15 reps), and dumbbell forward bend ( 3 sets, 15 reps each side). Phase five was when functional sport specific activities and standing balance were being introduced. The exercises were related to basketball by mimicking defensive moves, push offs on the ground, passing the ball, and different positions for shooting the ball. All exercises were performed while maintaining activation of the LPHC.

Phase 6 (Table C12): During the sixth phase, the subject performed multidirectional lunges with medicine ball twist ( 3 sets, 15 reps), uneven single leg squats ( 3 sets, 15 reps), Bosu squat with medicine ball twist ( 3 sets, 15 reps), dumbbell side bends ( 3 sets, 15 reps each side), unstable push-ups ( 3 sets, 15 reps), medicine ball slam ( 3 sets, 15 reps), and side step lunge with wood chop (3 sets, 15 reps). Phase six was a continuation of phase five with functional sport specific exercises with the exercises increasing in difficulty by adding uneven surfaces to challenge the balance of the subject. The exercises were related to basketball by mimicking defensive moves, push offs on the ground, passing, the ball, and different positions for shooting the ball while maintaining activation of the LPHC. 
Statistical Analysis

Descriptive analysis consist of means and standard deviations for pretest and posttest data for the SEBT, AFT, and SLRT. Six separate 1 x 2 repeated measures ANOVA were used to compare pretest and posttest results for the SEBT (anterior, posterolateral, and posteromedial directions) for the left and right leg. A 1 x 2 repeated measures ANOVA was used to compare pretest and posttest results for the AFT. Two $1 \times 2$ repeated measures ANOVA were used to compare pretest and posttest results for SLRT (anterior/posterior pelvic tilt) for each leg. The level of significance was set at $\mathrm{P}=.05$ for all analyses. No correction for multiple comparisons were made; instead Cohen $d$ measures of effect size were calculated based on the mean differences of test scores (pretest and posttest) divided by the reference SD (pretest) with corresponding 95\% confidence intervals (CI). The strength of effect sizes was determined as

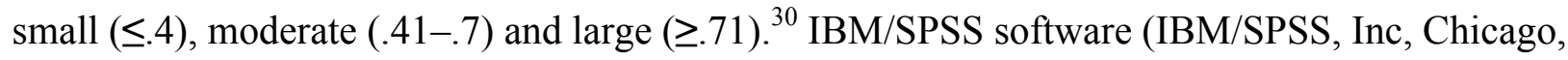
IL) version 24.0 was used for all analyses.

\section{RESULTS}

Single Leg Raise Test/Level Belt Pro

Descriptive statistics for the pre-test and post-test data of the Single Leg Raise Test/Level Belt Pro can be found in Table D1. There was a significant difference between pre- and post-test for the Single Leg Raise Test for the left leg $\left(F_{1,13}=7.556, P=0.017, E S=-0.80,95 \% C I=-1.57\right.$ to -0.03). The effect size is considered large. There was not a significant difference between preand post-test for the Single Leg Raise Test for the right leg $\left(\mathrm{F}_{1,13}=3.236, \mathrm{P}=0.095, \mathrm{ES}=-0.51\right.$, $95 \% \mathrm{CI}=-1.26-0.24)$. The effect size is considered moderate. 
Descriptive statistics for the pre-test and post-test data for the Abdominal Fatigue Test can be found in Table D1. There was not a significant difference between pre- and post-test for the abdominal fatigue test $\left(\mathrm{F}_{1,13}=3.418, \mathrm{P}=0.087, \mathrm{ES}=1.68,95 \% \mathrm{CI}=0.82-2.54\right)$. The effect size is considered large.

Star Excursion Balance Test

Descriptive statistics for the pre-test and post-test data for the Star Excursion Balance Test can be found in Table D1. There was not a significant difference between pre- and post-test for the Star Excursion Balance Test for the left leg in the anterior direction $\left(\mathrm{F}_{1,13}=0.586\right.$, $\mathrm{P}=0.458, \mathrm{ES}=-0.78,95 \% \mathrm{CI}=-1.55$ to -0.01$)$. The effect size is considered large. There was not a significant difference between pre- and post-test for the Star Excursion Balance Test for the left leg in the posteromedial direction $\left(\mathrm{F}_{1,13}=0.463, \mathrm{P}=0.508, \mathrm{ES}=-0.54,95 \% \mathrm{CI}=-1.30\right.$ to 0.21$)$. The effect size is considered moderate, but crossed 0 . There was not a significant difference between pre- and post-test for the Star Excursion Balance Test for the left leg in the posterolateral direction $\left(\mathrm{F}_{1,13}=0.365, \mathrm{P}=0.556, \mathrm{ES}=-0.48,95 \% \mathrm{CI}=-1.23-0.27\right)$. The effect size is considered moderate, but crossed 0 . There was not a significant difference between pre- and post-test for the Star Excursion Balance Test for the right leg in the anterior direction $\left(\mathrm{F}_{1,13}=0.753, \mathrm{P}=0.401, \mathrm{ES}=\right.$ $-1.02,95 \% \mathrm{CI}=-1.81$ to -0.23$)$. The effect size is considered large. There was not a significant difference between pre- and post-test for the Star Excursion Balance Test for the right leg in the posteromedial direction $\left(\mathrm{F}_{1,13}=0.559, \mathrm{P}=0.468, \mathrm{ES}=-0.73,95 \% \mathrm{CI}=-1.50\right.$ to 0.03$)$. The effect size is considered large. There was not a significant difference between pre- and post-test for the Star Excursion Balance Test for the right leg in the posterolateral direction $\left(\mathrm{F}_{1,13}=1.03, \mathrm{P}=0.328\right.$, $\mathrm{ES}=-1.15,95 \% \mathrm{CI}=-1.95$ to -0.35$)$. The effect size is considered large.

\section{DISCUSSION}


The purpose of this study was to determine if conducting a six week LPC and balance training program would increase LPC and balance in high school male basketball players. The results showed significant differences in the SLRT for the left leg with a large effect size showing the six-week intervention had an effect on LPC for the left leg. There was not a significant difference for the SLRT on the right leg, but the effect size was moderate which could indicate that six weeks may not be long enough to show results. No significant differences were found for the SEBT or AFT. The AFT showed a large effect size and a difference in means with an increase for post testing though no significant difference was found. One of two experimental hypothesis was accepted in this study as there was an increase in LPC after a six-week LPC and balance intervention as measured by the SLRT. As this study is the first to measure LPC and dynamic balance in boys' basketball players after a six-week LPC and balance intervention, known to the author, there are no other known significant findings that can be used for comparison

Lumbopelvic Control

The LPHC, also known as "the core", is the collection of anatomical structures surrounding the lumbar vertebrae, pelvic girdle, and hip joints (lumbopelvic-hip complex) along with the neuromuscular structures and fascial structures along those areas. "Providing the ability of the neuromuscular system to control and protect the lumbar spine and pelvis from abnormal movements in all three planes (frontal, sagittal, and transverse) and maintain a neutral position" is one of the main functions of the LPHC., 12-14, 31 Basketball players especially need to have a strong LPHC as basketball requires movement in multiple directions while ball handling, boxing out, rebounding, jump shots, and defensive positions all while putting the lumbar spine and pelvis in abnormal positions. The purpose of LPC in basketball is to maintain spine 
stabilization while performing those skills on the court. The activation of the LPHC musculature provides stiffness/stabilization around the central mass of the trunk and pelvis, which contributes stability to the center of gravity of the body. ${ }^{14}$ Kibler et al. ${ }^{14}$ further described the role of LPHC to provide "proximal stability for distal mobility". 4, 32, 33 As the LPHC remains in a stable, neutral position, the upper and lower extremities gain more functional movement and acceleration of movements. ${ }^{4,14,32,33}$ Despite only the left leg showing an increase in LPC, the gains evident on this side may have significant implications for basketball players that are right hand dominant.

All fourteen participants were right-handed and used the left leg as the lead/jumping leg. Continuously using the left leg as the take-off leg repeatedly in basketball games and practices, as the training program took place in-season, could be the reason why the left LPC improved. However, it was surmised that the true increase was related to the intervention as both the right and left legs were similar at baseline, but not at the end of the study. Further explanation could be related to the LPC intervention and the progression that was employed during the six-week program. The exercises used in this study were comprised of exercises that would be beneficial to the basketball player. All the exercises in this intervention protocol were taken from previous studies and chosen for their relationship with movements of basketball. ${ }^{5,11,15,22,26-29}$ All LPC exercises were progressed in phases when the previous phase was completed successfully. The exercises began with contraction and static hold of LPHC musculature to improve proper activation. ${ }^{15}$ The exercises progressed to more advanced contract and static hold in quadruped and standing positions. ${ }^{15}$ The program finally progressed to functional sport specific exercises while maintaining the activation of the $\mathrm{LPHC},{ }^{15}$ hence, the reason why the left LPC improved more so than the right. 
Lack of previous studies leaves little to provide a rationale why left side LPC increases in basketball players following an intervention. Studies that do exist examined the relationship between LPC and performance, but there was no indication that increasing only LPC will help improve performance. ${ }^{13,21,33,34}$ Studies show a decrease in injuries with increased LPC, which could be important during the lengthy basketball season. ${ }^{4,35,36}$

No significant difference was found in the AFT for this study though a large effect was shown along with an increase in the means compared pre-test to post-test. The means of the AFT (Table D1) showed an increase which should have shown some significant difference in the results, but the standard deviation was a result of one participant performing at a higher level than the other participants in the AFT. The AFT is also considered more of a LPHC endurance test though it can be used for measurements on LPHC stability as the test requires a constant contraction around the spinal column and hip complex. ${ }^{5,20}$ Bliven et al. ${ }^{5}$ stated that muscular endurance is more important than muscular strength for the purpose of LPC due to the constant contracture around the spine keeping it in a stable position. For this purpose, the test was used in this study in order to detect increases in LPC along with the SLRT in order to see if endurance (which is important to LPC) of the LPHC increased as well. The test is more static in nature while the exercises in the intervention were designed to be functional and dynamic for basketball players which could be why significant increases did not occur with the AFT. Exercises, such as the prone plank, bird dogs, push throughs, cross over crunch and medicine ball sit-up, used in phases 3 and 4 are designed to improve LPHC musculature strength as well as stabilization so the large effect size in the AFT could come from the use of these exercises. While increased muscular strength of the LPHC increased with the intervention, the intervention did not show an increase in LPC as there was no significant difference between pre/posttest. The reason is 
related to one individual holding the position during the posttest longer than the others. This outlier was depicted in the standard deviation.

Dynamic Balance

Balance is considered the ability to maintain the body's center of gravity over the base of support and results from neuromuscular actions in response to continuous visual, vestibular, and somatosensory feedback. ${ }^{6,9,11,37}$ There are two divisions of balance, static and dynamic. Dynamic balance is the ability to perform a task while maintaining a stable position. ${ }^{8,}{ }^{11}$ Dynamic balance has a greater relevance in athletics than static balance as it relates to stability during functional movement. ${ }^{8}$ Struzik et al. ${ }^{10}$ stated that basketball players exhibited a greater level of balance when compared to other sports due to the control over body and the ball simultaneously which relates to dynamic balance. Filipa et al.'s study ${ }^{17}$ concluded that "a neuromuscular training program that is focused on core stability, specifically the lower extremity strength, would affect the performance of an athlete in the Star Excursion Balance Test" since it assessed dynamic balance. $^{6}$

Boccolini et al. ${ }^{6}$ stated that the sport of basketball forces the individual player into various situations of balance instability, such as accelerations/decelerations, changes in direction, penetrations into the defensive perimeter, boxing out, dribbling, and defense position recovery, while receiving physical contact from other players on the court. Dynamic balance is important in athletics, especially basketball. ${ }^{8}$ Exercises that should be implemented in balance training programs should begin with stabilization of the spine and body on a stable ground. ${ }^{38}$ Difficulty in balance should be increased by introducing unstable surfaces to stance. ${ }^{38-40}$ Eyes can be closed to add difficulty to balance as it removes the visual stimulus that allows the central nervous system to stabilize the body. ${ }^{38,39}$ These exercises can be progressed by involving extra stimulus, such as 
throwing and receiving a ball, to help create more functional movement while maintaining balance. ${ }^{38,39}$ Progressions of single leg squats from even to uneven surfaces, squats on Bosu ball, and dynamic lunges were used in phases 5 and 6 for dynamic balance exercises. In this study, SEBT scores did not improve from pre-test to post-test despite all subjects completing the six phases successfully during the six-week intervention. In order to benefit from dynamic balance, more exercises should be included, such as more balance stabilization progressions earlier in the intervention, or the intervention should be extended in order to incorporate more exercises to help with dynamic balance improvement.

The SEBT can be used in the basketball player to detect deficits in dynamic balance. Unfortunately, the results from this study actually showed that the reach distances decreased for all 3 directions on both the right and left leg. At first it could be determined that this was related to the lack of dynamic balance exercises included in the intervention protocol. But in actuality, this was related to the lack of effort during the post-testing procedures. The lack of motivation could have come from a losing basketball season and not wanting to stay after school once the season was over. Another factor in decreased post-testing results could be fatigue from a long basketball season. Practices, along with games, lasted six days a week which could have contributed to muscular fatigue, especially since no other conditioning programs were implemented during the season. Flexibility could have played a role in decreased scores, especially for the anterior and posteromedial directions for the SEBT. Although a daily stretching routine for flexibility was used with dynamic and static stretching techniques, the muscles involved with ankle dorsiflexion (i.e. anterior tibialis) were not acknowledged in those stretching routines. It is plausible that muscle tightness could have caused a decrease in range of motion of ankle dorsiflexion, which can lead to decreased SEBT scores. Lastly, although, not 
evaluated in this study, the type of shoe the athlete was wearing during testing could have affected scores as some high top basketball shoes can limit ankle range of motion. In addition, different shoes could have been worn during pre and post-testing as it was not stipulated as to what shoes to wear.

Despite the possibility of lack of effort, fatigue, and flexibility, the right leg in the anterior, posteromedial, and the posterolateral directions and the left leg anterior direction had a large effect size, while the posteromedial and posterolateral directions on the left leg had moderate effect sizes. Perhaps this can be attributed to the sport of basketball where movements are more common in all directions away from the pivot leg (right leg) especially in the lateral directions. The right leg (when the left leg is established as the lead leg) is used as the stabilizing leg for motions when the left leg is the reach leg.

Filipa et al. ${ }^{17}$ and Notarnicola et al. ${ }^{9}$ used longer intervention programs (eight and twelve weeks, respectively) for their studies when using the SEBT to measure dynamic balance after administration of an intervention program. Notarnicola et al. ${ }^{9}$ used a training session intervention three times a week for twelve weeks. Filipa et al. ${ }^{17}$ used a neuromuscular training program with LPC and lower body strength exercises in order to increase dynamic balance. Filipa et al.'s study ${ }^{17}$ differs from this study by the types of exercises used. Filipa et al.'s study ${ }^{17}$ began with more functional and dynamic exercises to improve LPC and also added lower extremity strength exercises. This study focused on LPC and the ability to maintain control during the first phases of the intervention with emphasis on activation of the musculature of the LPHC with dynamic balance exercises being introduced in phases 5 and 6 . The emphasis of LPHC activation at the beginning of the intervention helped to improve the proximal stability of the body with 
improvements in LPC, but the exercises did not help with improvements in distal stability to increase dynamic balance.

Clinical Importance

The strength of this study lies in the clinical importance of these findings. This study was able to show increases in LPC, specifically in the left leg, after a six-week LPC and balance intervention program. Increases in LPHC are important for the sport of basketball as studies conducted by Chaudhari et al., ${ }^{4}$ Fredericson et al.,${ }^{35}$ and Leetun et al. ${ }^{36}$ have shown a decreases in injuries. The increases in LPC were caused by selection of LPC exercises. The exercises in the intervention focused on improving activation of the LPHC musculature before moving on to functional activities that could improve LPC and balance for the athlete. Exercise phases could be extended time-wise in order to further create more difficult phases. More balance exercises, such as single leg progressions with stable and unstable surfaces, could be added to the intervention in order to show better improvements in dynamic balance as this study did not show improvement despite moderate and some large effect sizes. The types of exercises and the duration spent on completion of the exercises administered can affect what increases within the study. For example, LPC increases were more evident as the early phases included more LPHC exercises. Thus, in order to improve balance, dynamic balance exercises should be added to earlier phases.

The tests used in this study also have clinical importance as the clinician may choose to use these tests to evaluate LPC and dynamic balance. The SLRT has shown to be a reliable test for LPC when being used by one rater for determining improvements of pelvic tilt. ${ }^{19}$ The SLRT can be useful in the clinical setting as it is an easy test for the athlete to conduct as they perform the simple action of lifting their leg while the CoreX belt is around their waist. ${ }^{4}$ It is easier to 
perform the SLRT versus the AFT as the AFT requires more effort to hold the sit-up position where the SLRT just requires the athlete to raise their leg. The SEBT would be the most difficult to perform in a clinical setting, not in terms of set-up as the test requires a grid to be set-up with measuring tapes, but with exertional effort from the athlete to perform each direction three times. $^{23}$ The test has shown to be very reliable to test dynamic balance, but it can be the most complicated to perform. ${ }^{23}$

Limitations of Study

The number of participants used was a limitation as there were only 21 boys' basketball players to begin with. Six players were excluded from the study and one quit the team before pre-testing could begin. Of the fourteen who completed pre-testing, one sustained a hand fracture during the time of the fifth phase of the intervention and was unable to finish the study; another player completed the six phases of the intervention, but did not complete the post-testing for data collection. The size of the sample was also a limitation, as the sample was of convenience and limited to only one high school boys' basketball team. As only teenage males were used, some of whom could still be growing or undergoing a growth spurt throughout the period of this study may have had an effect on the SEBT. However, reach distances were normalized to leg length, which may have counterbalanced this concern. A control group of basketball athletes was not included to evaluate the intervention, but moderate to large effect sizes were evident. To accommodate not having a control group both legs were tested for the Single Leg Raise test and the SEBT. However, further investigation with a control group may be warranted to verify the changes. The order the tests were completed could be a limitation as the left leg was continuously used as the first leg tested which could result in better scores for the left leg compared to the right leg. The biggest limitation of performing the study came during post- 
testing collection due to motivation of the athletes. Post-testing occurred during the week after the season had completed. With the season completed, the players lacked motivation to come in after school to complete the tests. They also lacked motivation to complete the tests to the best of their ability.

\section{CONCLUSION}

There was a significant difference with the SLRT on the left leg after the completion of a six-week LPC and balance intervention program. There were no significant differences with the SLRT on the right leg, AFT, or any directions of the SEBT. The SLRT on the right leg, SEBT left anterior, SEBT left posteromedial, SEBT left posterolateral, and SEBT right posteromedial all showed moderate effect sizes while the AFT, SEBT right anterior, and SEBT right posterolateral showed large effect sizes. While there were no significant differences, there was an increase in mean of scores of the AFT from pre-test to post-test following the intervention program. All SEBT directions on both legs showed a decrease in mean of scores from pre-test to post-test. The six-week LPC and balance intervention program could be used to increase LPC, but do not show an ability to improve dynamic balance. These results support the use of a sixweek LPC and balance intervention to improve LPC in clinical practice. 


\section{REFERENCES}

1. Estimated probability of competing in athletics beyond the high school interscholastic level. NCAA Research. 2013. http://www.ncaa.org/sites/default/files/Probability-ofgoing-pro-methodology_Update2013.pdf

2. Borowski LA, Yard EE, Fields SK, Comstock RD. The epidemiology of us high school basketball injuries, 2005-2007. Amer J Sport Med. 2008;36(12):2328-2335.

3. Plisky PJ, Rauh MJ, Kaminski TW, Underwood FB. Star excursion balance test as a predictor of lower extremity injury in high school basketball players. J Orthop Sports Phys Ther. 2006;36(12):911-919.

4. Chaudhari AM, Mckenzie CS, Pan X, Oñate JA. Lumbopelvic control and days missed because of injury in professional baseball pitchers. Am J Sports Med. 2014;42(11):273440 .

5. Bliven $\mathrm{KCH}$, Anderson BE. Core stability training for injury prevention. Sports Health. 2013;5(6):514-522.

6. Boccolini G, Brazzit A, Bonfanti L, Alberti G. Using balance training to improve the performance of youth basketball players. Sport Sci Health. 2013;9(2):37-42.

7. Bishop RD, Hay JG. Basketball: the mechanics of hanging in the air. Med Sci Sports. 1979;11(3):274-277.

8. Ricotti L. Static and dynamic balance in young athletes. J Human Sport Exer. 2011;6(4):616-628.

9. Notarnicola A, Maccagnano G, Tafuri S, Pesce V, Digiglio D, Moretti B. Effects of training on postural stability in young basketball players. Muscle Ligaments Tendons J. 2015;5(4):310-315.

10. Struzik A, Pietraszewski B, Zawadzki J. Biomechanical analysis of the jump shot in basketball. J Hum Kinet. 2014;42:73-79.

11. Yaggie JA, Campbell BM. Effects of balance training on selected skills. J Strength Cond Res. 2006;20(2):422-428.

12. Hodges PW. Core stability exercise in chronic low back pain. Ortho Clin N Amer. 2003;34(2):245-254.

13. Mills JD, Taunton JE, Mills WA. The effect of a 10-week training regimen on lumbopelvic stability and athletic performance in female athletes: A randomized-controlled trial. Phy Ther Sport. 2005;6(2):60-66. 
14. Kibler WB, Press J, Sciascia A. The role of core stability in athletic function. Sports Med. 2006;36(3):189-198.

15. Rivera CE. Core and lumbopelvic stabilization in runners. Phys Med Rehabil Clin N Am. 2016;27(1):319-337.

16. Barr KP, Griggs M, Cadby T. Lumbar stabilization: core concepts and current literature, Part 1. Am J Phys Med Rehabil. 2005;84(6):473-480.

17. Filipa A, Byrnes R, Paterno MV, Myer GD, Hewett TE. Neuromuscular training improves performance on the star excursion balance test in young female athletes. $J$ Orthop Sports Phys Ther. 2010;40(9):551-558.

18. Chaudhari AM, Mckenzie CS, Borchers JR, Best TM. Lumbopelvic control and pitching performance of professional baseball pitchers. J Strength Cond Res. 2011;25(8):21272132.

19. Yedimenko J, Jamison ST, McNally MP, McKenzie CS, Onate JA, Chaudhari, AM. Intra- and inter-rater reliability of a single-leg raise test of pelvic sagittal control. Paper presented at: American College of Sports Medicine Annual Meeting; May 29, 2013; Indianapolis, IN.

20. McGill SM, Childs A, Liebenson C. Endurance times for low back stabilization exercises: clinical targets for testing and training from a normal database. Arch Phys Med Rehabil. 1999;80(8):941-944.

21. Nesser TW, Huxel KC, Tincher JL, Okada T. The relationship between core stability and performance in division I football players. J Strength Cond Res. 2008;22(6):1750-1754.

22. Brumitt J, Matheson JW, Meira EP. Core stabilization exercise prescription, part I: current concepts in assessment and intervention. Sports Health. 2013;5(6):504-509.

23. Hyong IH, Kim JH. Test of intrarater and interrater reliability for the star excursion balance test. J Phys Ther Sci. 2014;26(8):1139-1141

24. Gribble PA, Kelly SE, Refshauge KM, Hiller CE. Interrater reliability of the star excursion balance test. J Ath Train. 2013;48(5):621-626.

25. Gribble PA, Hertel J. Considerations for normalizing measures of the star excursion balance test. Measure Phys Edu Exer Sci. 2003;7(2):89-100.

26. Akuthota V, Ferreiro A, Moore T, Fredericson M. Core stability exercise principles. Curr Sports Med Rep. 2008;7(1):39-44.

27. Mcgill SM. Low back exercises: evidence for improving exercise regimens. Phys Ther. 1998;78(7):754-765. 
28. Mcguine TA, Keene JS. The effect of a balance training program on the risk of ankle sprains in high school athletes. Am J Sports Med. 2006;34(7):1103-1111.

29. Dale RB, Lawrence R. Principles of Core Stabilization for Athletic Populations. Athl Ther Today. 2005;10(4):13-18.

30. Mckeon PO, Ingersoll CD, Kerrigan DC, Saliba E, Bennett BC, Hertel J. Balance training improves function and postural control in those with chronic ankle instability. Med Sci Sports Exerc. 2008;40(10):1810-1819.

31. Hill J, Leiszler M. Review and role of plyometrics and core rehabilitation in competitive sport. Curr Sports Med Rep. 2011;10(6):345-351.

32. Willson JD, Dougherty CP, Ireland ML, Davis IM. Core stability and its relationship to lower extremity function and injury. J Am Acad Orthop Surg. 2005;13(5):316-25.

33. Okada T, Huxel KC, Nesser TW. Relationship between core stability, functional movement, and performance. J Strength Cond Res. 2011;25(1):252-261.

34. Sato K, Mokha M. Does core strength training influence running kinetics, lowerextremity stability, and 5000-m performance in runners? J Strength Condition Res. 2009;23(1):133-140.

35. Fredericson M, Moore T. Muscular balance, core stability, and injury prevention for middle- and long-distance runners. Phys Med Rehabil Clin N Am. 2005;16(3):669-689.

36. Leetun DT, Ireland ML, Willson JD, Ballantyne BT, Davis IM. Core stability measures as risk factors for lower extremity injury in athletes. Med Sci Sports Exerc. 2004;36(6):926-934.

37. Blackburn T, Guskiewicz KM, Petschauer MA, Prentice WE. Balance and joint stability: the relative contributions of proprioception and muscular strength. J Sport Rehabil. 2000;9(4):315-328.

38. Cruz-diaz D, Lomas-vega R, Osuna-pérez MC, Contreras FH, Martínez-amat A. Effects of 6 weeks of balance training on chronic ankle instability in athletes: a randomized controlled trial. Int J Sports Med. 2015;36(9):754-60.

39. Gioftsidou A, Malliou P, Pafis G, Beneka A, Godolias G, Maganaris CN. The effects of soccer training and timing of balance training on balance ability. Eur J Appl Physiol. 2006;96(6):659-664.

40. Aggarwal A, Zutshi K, Munjal J, Kumar S, Sharma V. Comparing stabilization training with balance training in recreationally active individuals. Int J Ther Rehab. 2010;17(5):244-253. 


\section{APPENDICES}




\section{APPENDIX A}

\section{THE PROBLEM}

\section{Research Question}

Basketball has become a very popular sport in the United States. Many adolescents participate in this sport with dreams of playing at the college and professional level. With the sport becoming increasingly popular, athletes are looking for different strength and conditioning programs that will help provide a competitive edge to make it in the National Basketball Association (NBA). The issue with most of the strength and conditioning programs that are available to these athletes is that there is limited research conducted on the validity of these programs. Two increasingly popular strength and conditioning programs for basketball players are core, also known as lumbopelvic control (LPC) and balance.

Research conducted on LPC is quite varied in the athletic population as each study incorporates anatomical structures based on an individual definition instead of a standardized one. ${ }^{40}$ Usually postural control and load forces placed on the body are considered, ${ }^{12}$ using the focus of the neuromuscular system to control and protect the lumbar spine and pelvis from abnormal movements in all three planes (frontal, sagittal, and transverse) and maintain a neutral

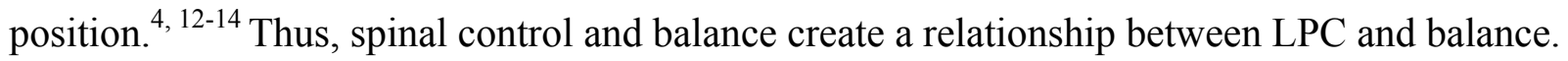
To incorporate this concept into a training program, a spine neutral position is needed to be able to maintain the body over the center of gravity. ${ }^{8}$ Bishop and $\mathrm{Hay}^{7}$ hypothesized that the center of gravity moves in correlation to the athlete's trunk. When an athlete, especially a basketball athlete, maintains a spine neutral position the trunk would be in a neutral position causing the body to equally distribute weight over the center of gravity during jump shots, defensive stance, and free throws. ${ }^{7,8}$ A spine neutral position is needed to properly perform basketball skills such 
as accelerations and decelerations on the court, change of direction, penetrations into the defensive perimeter, boxing out, dribbling, and defensive position recovery. ${ }^{6,8}$ Therefore, an increase in LPC is important to control and maintain the lumbar spine and pelvis to maintain the spine neutral position. Thus, a spine neutral position is better maintained and controlled by increasing the amount of LPC the athlete has.

Balance is considered "the ability to maintain the body's center of gravity over the base of support and results from neuromuscular actions in response to continuous visual, vestibular, and somato-sensory feedback". ${ }^{6}$ An athlete must maintain an equal amount of weight surrounding the center of gravity in order to maintain balance. ${ }^{6}$ A disequilibrium of the athlete could occur with many movements including twisting movement of the feet, jump shots, and offensive/defensive rebounds. ${ }^{6,9}$ Players also need to have not only an awareness of the body, but of the ball as well. ${ }^{10} \mathrm{~A}$ disruption in the equilibrium could cause a disruption in the athlete's performance by the unequal balance of weight over the center of gravity throwing the athlete off balance. ${ }^{6,10}$ Balance plays a crucial role in everyday activities such as walking and running. ${ }^{8,9}$ It also plays a crucial role in all sport activities, ${ }^{9}$ especially basketball players. ${ }^{10}$ Though there is not sound evidence to show an increase in balance can increase basketball skills; there is enough research to show that balance plays a crucial role in the sport of basketball when performing certain skills. $^{11}$

Thus, it can be seen that LPC and balance are important for the basketball athlete. However, little guidance has been provided in the literature for what constitutes a training program of this nature. In baseball, LPC training program studies were conducted in order to detect a decrease in injuries when LPC is increased. ${ }^{4}$ Filipa et al., ${ }^{17}$ used female soccer players who underwent a neuromuscular training program with core stability exercises to see if 
performance of the star excursion balance test (SEBT) would improve. ${ }^{17}$ Performing a neuromuscular training program with a focus on core stability and strength exercises improved reach distances on the SEBT. ${ }^{17}$ Unfortunately, there is no research relating these results to high school basketball to the author's knowledge, thus, more research should be established on LPC and balance training for high school basketball players. Because there is limited information, and core and balance are necessary for basketball athletes, there is a need for this study.

Thus, the following research question is asked: Does performing a LPC and balance intervention program for six weeks help increase LPC and dynamic balance in male high school basketball players?

Experimental Hypothesis

1. There will be an increase in LPC as measured by the SLRT and the AFT from baseline after a six week LPC and balance intervention is administered.

2. There will be an increase in dynamic balance in the anterior direction more than the posterolateral and posteromedial directions from baseline after a six week LPC and balance intervention is administered.

Assumptions

1. Subjects in this experiment will meet inclusion criteria.

2. The Level Belt Pro application and tape measure for Star Excursion Balance Test used are valid and reliable for use in this study.

3. Single leg raise test and abdominal fatigue test used are valid and reliable.

4. Evaluation tests will be performed identically on subjects.

5. Pre and post-tests will be performed to exact specifications.

6. Each subject will perform the exercises assigned correctly.

7. All exercises intervention will be completed twice a week for six weeks.

Delimitations 
1. Subject population will be limited to a small sample of male basketball athletes from one high school in western Pennsylvania.

2. Subjects will be between the ages of 15 to 18 and will only participate in high school basketball at the time of the study.

Operational Definitions

1. Balance- The ability to maintain the body's center of gravity over the base of support and results from neuromuscular actions in response to continuous visual, vestibular, and somato-sensory feedback ${ }^{6}$

2. Dynamic balance- Ability to perform a task while maintaining a stable position ${ }^{8}$

3. Lumbo-pelvic hip complex (LPHC)- Referred to as the "core"; central nervous system, the lumbar vertebrae, the pelvic girdle, hip joints, thorcolumbar fascia, rectus abdominis, transverse abdominis, erector spinae, multifidus, gluteus maximus, gluteus medius, gluteus minimus, quadratus laborum, adductor magnus, adductor longus, adductor brevis, pectineus, superior/inferior gemelli, quadratus femoris, and obturator externus/internus ${ }^{4}$, $14,29,35$

4. Lumbopelvic control (LPC)- Referred to as "core stability"; the ability of the neuromuscular system to control and protect the lumbar spine and pelvis from abnormal movements in all three planes (frontal, sagittal, and transverse) and maintain a neutral position. $^{4,12-14}$

5. Level Belt Pro Application- Also referred to as An iPod-based tilt sensor; which measures core stability (also known as LPC), anterior/posterior pelvic tilt along with lateral pelvic tilt to help determine neutral pelvic tilt. ${ }^{18}$

6. Level Belt test- Referred to as the Single Leg Raise Test (SLRT), which measures neutral position of pelvic tilt with Level Belt Pro App in a functional manner to determine core $(\mathrm{LPC})^{18}$

7. Pelvic tilt- Motion of the pelvic girdle in an anterior, posterior, or lateral movement away from a neutral position ${ }^{27}$

8. Static balance- Maintenance of a base of support with minimal to no movement ${ }^{8}$

9. Star Excursion Balance Test (SEBT)- Functional balance test that measures dynamic balance in the anterior, posteromedial, and posterolateral direction. ${ }^{42}$

10. Trunk flexor test- Measures functional core stability and is also referred to as the abdominal fatigue test (lumbo-pelvic complex control) ${ }^{5}$ 


\section{Limitations}

1. No gold standard measurement of lumbo-pelvic complex control.

2. A universal definition of lumbo-pelvic complex or lumbo-pelvic complex control has been created for generalizable use.

3. The Level Belt Pro Application only measures in one plane: the sagittal plane.

4. Subjects may not give $100 \%$ while performing exercises and performing evaluation tests.

5. Subjects may drop out at any time during the duration of this study.

6. Only three directions are measured in modified star excursion balance test: anterior, posteromedial, and posterolateral.

7. There is no control group.

8. Limited research concerning lumbopelvic control related directly to basketball.

Significance of Study

Core training, which is related to LPC, and balance training are becoming popular strength and conditioning trends. Research should be conducted on how these training programs will improve LPC and balance in athletics, specifically basketball. Research has been conducted on speed, endurance, shooting, and jump height in basketball, but lacks specific training program protocols that will improve LPC and balance. The benefits of this study will provide a protocol that incorporates LPC and balance intervention to improve LPC and balance in high school basketball players. Future researchers should be able to apply the results to conduct additional studies in basketball. Interventions used for this study can be used in future research by expanding the sample size and diversity to create a more reliable study.

This study is being conducted to provide basketball coaches, strength and conditioning coaches, and athletes evidence-based training protocols to implement into strength and conditioning routines. The results of this study can be disseminated through publications, 
symposiums, workshops, or conventions to provide information about the exercises that should be incorporated into a basketball LPC and balance training program. 


\section{APPENDIX B}

\section{LITERATURE REVIEW}

Introduction

Basketball is one of the most popular sports in the United States at the high school, college, and professional levels. ${ }^{44,45}$ Based on this, strength and conditioning training for basketball has become a popular trend in order to move to the next level of performance (i.e. college or pro). Lumbopelvic control (LPC) and balance training have become very popular topics within the strength and conditioning realm. However, evidence based or best clinical practice research is very limited in what should be incorporated into a LPC and balance training program, especially for high school basketball players.

Lumbopelvic-hip complex (LPHC) and LPC have multiple definitions and involve varied terminology depending on the context of which it is being used. These terms are used interchangeably with the terms core and core stabilization. ${ }^{4,5,31,35,41,45}$ The purpose of LPC in basketball is to maintain spine stabilization while performing multiple skills on the court. Another key component of basketball is balance. Balance is essential for all daily activities and sport activities. ${ }^{9,42}$ It helps with many skills on the court as the basketball player must run, jump, and spin all while handling the ball. ${ }^{46}$ The relationship that has not been studied is what type of training program should be incorporated that included LPC and balance. Both involve stabilization against internal and external perturbations, and in order to reduce abnormal perturbations, which would cause disequilibrium, the LPHC needs to be strengthened to improve stabilization. 6, 9, 17,46 This literature review will discuss lumbopelvic-hip complex anatomy, lumbopelvic control and what it entails, measurements of lumbopelvic control, balance, 
measurements of balance and intervention programs for LPC and balance. All of these will be discussed in their relevance to basketball.

Lumbopelvic-Hip Complex (LPHC) Anatomy

The anatomical structures that comprise the LPHC, also known as "the core", vary from researcher to researcher due to disagreement of which muscles and other anatomical structures actually make up the LPC. 4, 5,31,35,41, 45 The reason for this uncertainty is how researchers think the LPHC will activate and what motions will be created. Some researchers will claim the LPHC consists of all the musculature from the upper spinal region down to the hamstrings and quadriceps. ${ }^{14,35}$ Though other researchers will claim only the musculature from the trunk and pelvis regions should be considered as LPHC. ${ }^{14,35}$ Another difference between research studies that creates inconsistencies in the anatomical definition of LPHC is the inclusion of neurological and fascial structures. Some researchers will talk in depth about the involvement of the central nervous system and other neuromuscular elements; others will only discuss the passive and active structures of the LPHC. ${ }^{14,15,35}$ For the purpose of this study, the anatomical structures consist of the structures surrounding the lumbar vertebrae, pelvic girdle, and hip joints (lumbopelvic-hip complex) along with neuromuscular structures and fascial structures. ${ }^{4}$ These structures are important in basketball as the structures of the lumbar spine and pelvic girdle provide control in many basketball activities such as jump shots, rebounds, accelerations/decelerations. $^{46}$

There are separate elements that work together to control the LPHC: neuromuscular control, passive subsystem, and active subsystem. ${ }^{5,16,26}$ The most important component of neuromuscular control is the central nervous system (CNS). ${ }^{12}$ Neuromuscular control refers to the body's nervous system having control over the muscle activation during movement. ${ }^{37,47}$ The 
CNS controls the activation of the musculature in the body, and when the musculature is affected by the sensation of internal or external forces (afferent system) then the CNS can send messages for the musculature to adjust (efferent system). ${ }^{5,12,13,15}$ Neuromuscular control is important in stabilizing the spine as it will send out signals to the musculature of the LPHC to activate in the case of internal or external perturbations acting against the spine. ${ }^{12,13,47}$ The passive subsystem consists of the static structures of the lumbar vertebrae, intervertebral discs, ligaments, and joint capsules. ${ }^{5,16,26}$ The importance of the passive subsystem is to absorb the forces placed on the structures to maintain static stabilization. ${ }^{5,15,16}$ The active subsystem consists of all the musculature of the LPHC. Every muscle in the LPHC along with origin, insertion, and action are listed in Table B1. These three subsystems must work together to maintain the stability of the LPHC with the neural system maintaining the control over the movements of the passive and active systems. 5,16

The different muscles can be categorized into two groups: local stabilizers and global stabilizers. ${ }^{5,12,13,48}$ The muscles that make up these two groups remain consistent amongst the literature. The local stabilizers contribute to stabilization of the spine and are located deep attaching to the lumbar vertebrae, consisting of the multifidus and transverse abdominis. $5,12,13,16$, ${ }^{48}$ The local stabilizers are dependent on the position of the body and controls the lumbar spine. ${ }^{5,}$

${ }^{15}$ The global stabilizers are the prime movers of the LPHC connecting the trunk to the extremities consisting of the erector spinae, quadratus laborum, and rectus abdominis. $5,12,13,16,48$ The global stabilizers distribute the forces equally amongst the body. ${ }^{12,15}$ The transverse abdominis and multifidus are widely agreed upon as important structures for LPC due to activation of these muscles results in the greatest stabilization of the spine. ${ }^{12,14,26,41}$ The multifidus helps provide stability as one of the local stabilizers by controlling the intervertebral 
stability. ${ }^{12}$ McGill et al. ${ }^{30,49}$ conducted a study showing the multifidi did not change in length throughout the entire lumbar range of motion indicating their major role in stabilization versus movement. Arokoski et al. ${ }^{48}$ conducted a study on the muscle activation patterns of the abdominal and paraspinal muscles during therapeutic exercises and limb movements. They showed muscle activation increased by performing certain therapeutic exercises and limb movements, particularly those involving hyperextension of the back. They were able to show that the contribution of the local stabilizers, multifidus and transverse abdominis, was specifically in stabilization of the spine. ${ }^{48}$ The global stabilizers were found to only provide trunk motion and did not contribute to stabilization. ${ }^{48}$ McGill et al. ${ }^{50}$ used EMG to indicate muscle activation in therapeutic exercises in order to determine proper progression of stabilization exercises. McGill et al. ${ }^{50}$ took progressions of the curl-up, side bridge, and bird dog exercises for the increased activation in order to show improvement in progression. Drysdale et al. ${ }^{51}$ used EMG to determine the activation of rectus abdominis and external oblique abdominis during the abdominal hollowing exercises. It was shown that the two global stabilizers show little activation during abdominal hollowing exercises causing the local stabilizers to independently work. ${ }^{51}$ Muscle co-activation of the anterior and posterior muscles of the LPHC is important as it will increase the intra-abdominal pressure and trunk stiffness which helps increase stability. ${ }^{35,41,52}$ Cholewicki et al. ${ }^{53}$ conducted a study using surface electromyography (EMG) in order to determine co-activation of the external oblique, internal oblique, rectus abdominis, multifidus, lumbar erector spinae, and thoracic erector spinae for spinal stability. The muscles provided proper stability in healthy individuals and that increased mass would improve the stability. ${ }^{53}$ The transverse abdominis co-activates with the pelvic floor muscles to increase the intra-abdominal pressure. ${ }^{26,52,54}$ The transverse abdominis is the most important in regards to intra-abdominal 
pressure as activation of the transverse abdominis can increase intra-abdominal pressure when it activates and maintains spine neutral. ${ }^{15}$ Intra-abdominal pressure is important as it contributes to increasing the stiffness of the spine. ${ }^{12,14}$ The thoracolumbar fascia is important as it connects around the entire trunk and creates connections between the upper and lower extremities. ${ }^{13,14,26}$ The thoracolumbar fascia attaches directly to the transverse abdominis and contributes to the stabilization of the spine and increasing intra-abdominal pressure when the transverse abdominis is activated. ${ }^{12,14}$ When intra-abdominal pressure and thoracolumbar tension are increased, the transverse abdominis provides increased stiffness to the intervertebral joints. ${ }^{12,}{ }^{14}$ The diaphragm also contributes to increasing intra-abdominal pressure. ${ }^{30}$

$\underline{\text { Table B1. Muscle Origin/Insertion/Action of the Lumbopelvic-hip complex }}{ }^{30,35}$

\begin{tabular}{|c|c|c|c|c|}
\hline Muscles & Origins & Insertions & Actions & Innervation \\
\hline Rectus Abdominis & $\begin{array}{l}\text { Pubic crest, pubic } \\
\text { symphysis }\end{array}$ & $\begin{array}{l}\text { Cartilage of fifth, } \\
\text { sixth, and seventh } \\
\text { ribs and xiphoid } \\
\text { process }\end{array}$ & $\begin{array}{l}\text { Flex vertebral } \\
\text { column, tilt pelvis } \\
\text { posteriorly }\end{array}$ & $\begin{array}{l}\text { T5-T12, ventral } \\
\text { rami }\end{array}$ \\
\hline Transverse Abdominis & $\begin{array}{l}\text { Lateral inguinal } \\
\text { ligament, iliac } \\
\text { crest, } \\
\text { thoracolumbar } \\
\text { fascia, and internal } \\
\text { surface of lower six } \\
\text { ribs }\end{array}$ & $\begin{array}{l}\text { Abdominal } \\
\text { aponeurosis to linea } \\
\text { alba }\end{array}$ & $\begin{array}{l}\text { Compress } \\
\text { abdominal contents }\end{array}$ & $\begin{array}{l}\text { T7-T12, } \\
\text { iliohypogastric } \\
\text { and ilioguinal, } \\
\text { ventral } \\
\text { divisions }\end{array}$ \\
\hline Quadratus Lumborum & Posterior iliac crest & $\begin{array}{l}\text { Last rib and } \\
\text { transverse processes } \\
\text { of first through } \\
\text { fourth lumbar } \\
\text { vertebrae }\end{array}$ & $\begin{array}{l}\text { Laterally tilt the } \\
\text { pelvis; Laterally } \\
\text { flex the vertebral } \\
\text { column to the same } \\
\text { side; Assist to } \\
\text { extend the vertebral } \\
\text { column; Fix the last } \\
\text { rib during forced } \\
\text { inhalation and } \\
\text { exhalation }\end{array}$ & $\begin{array}{l}\text { Lumbar plexus } \\
\mathrm{T} 12, \mathrm{~L} 1-3\end{array}$ \\
\hline $\begin{array}{l}\text { Erector Spinae (Spinalis, } \\
\text { Longissimus, } \\
\text { Iliocostalis) }\end{array}$ & $\begin{array}{l}\text { Common tendon } \\
\text { (thoracolumbar } \\
\text { aponeurosis) that } \\
\text { attaches to the } \\
\text { posterior surface of } \\
\text { sacrum, iliac crest, } \\
\text { spinous processes } \\
\text { of the lumbar and }\end{array}$ & $\begin{array}{l}\text { Various attachments } \\
\text { at the posterior ribs, } \\
\text { spinous and } \\
\text { transverse processes } \\
\text { of thoracic and } \\
\text { cervical vertebrae } \\
\text { and mastoid process } \\
\text { of temporal bone }\end{array}$ & $\begin{array}{l}\text { Laterally flex } \\
\text { vertebral column to } \\
\text { the same side; } \\
\text { extend vertebral } \\
\text { column }\end{array}$ & Spinal \\
\hline
\end{tabular}




\begin{tabular}{|c|c|c|c|c|}
\hline & $\begin{array}{l}\text { last two thoracic } \\
\text { vertebrae }\end{array}$ & & & \\
\hline Gluteus Maximus & $\begin{array}{l}\text { Coccyx, edge of } \\
\text { sacrum, posterior } \\
\text { iliac crest, } \\
\text { sacrotuberous and } \\
\text { sacroiliac ligaments }\end{array}$ & $\begin{array}{l}\text { Iliotibial tract and } \\
\text { gluteal tuberosity }\end{array}$ & $\begin{array}{l}\text { Extend the hip; } \\
\text { Laterally rotate the } \\
\text { hip; Abduct the hip }\end{array}$ & $\begin{array}{l}\text { Inferior gluteal } \\
\mathrm{L} 5, \mathrm{~S} 1, \mathrm{~S} 2\end{array}$ \\
\hline Gluteus Medius & $\begin{array}{l}\text { Gluteal surface of } \\
\text { ilium, between } \\
\text { posterior and } \\
\text { anterior gluteal } \\
\text { lines, just below the } \\
\text { iliac crest }\end{array}$ & $\begin{array}{l}\text { Lateral aspect of } \\
\text { greater trochanter }\end{array}$ & $\begin{array}{l}\text { Abduct hip } \\
\text { Anterior fibers: } \\
\text { Flex the hip; } \\
\text { Medially rotate the } \\
\text { hip } \\
\text { Posterior fibers: } \\
\text { Extend the hip; } \\
\text { Laterally rotate the } \\
\text { hip }\end{array}$ & $\begin{array}{l}\text { Superior gluteal } \\
\text { L4, L5, S1 }\end{array}$ \\
\hline Gluteus Minimus & $\begin{array}{l}\text { Gluteal surface of } \\
\text { the ilium between } \\
\text { the anterior and } \\
\text { inferior gluteal } \\
\text { lines }\end{array}$ & $\begin{array}{l}\text { Anterior aspect of } \\
\text { greater trochanter }\end{array}$ & $\begin{array}{l}\text { Abduct the hip; } \\
\text { Medially rotate the } \\
\text { hip; Flex the hip }\end{array}$ & $\begin{array}{l}\text { Superior gluteal } \\
\text { L4, L5, S1 }\end{array}$ \\
\hline Multifidus & $\begin{array}{l}\text { Sacrum and } \\
\text { transverse } \\
\text { processes of lumbar } \\
\text { through cervical } \\
\text { vertebrae }\end{array}$ & $\begin{array}{l}\text { Spinous processes of } \\
\text { lumbar vertebrae } \\
\text { through second } \\
\text { cervical vertebra }\end{array}$ & $\begin{array}{l}\text { Rotate the vertebral } \\
\text { column to the } \\
\text { opposite side; } \\
\text { Extend the } \\
\text { vertebral column }\end{array}$ & Spinal \\
\hline Adductor Magnus & $\begin{array}{l}\text { Inferior ramus of } \\
\text { the pubis, ramus of } \\
\text { ischium and ischial } \\
\text { tuberosity }\end{array}$ & $\begin{array}{l}\text { Medial lip of linea } \\
\text { aspera and adductor } \\
\text { tubercle }\end{array}$ & $\begin{array}{l}\text { Adduct the hip; } \\
\text { Medially rotate the } \\
\text { hip }\end{array}$ & $\begin{array}{l}\text { Obturator L2-4 } \\
\text { and Sciatic L4, } \\
\text { L5, S1 }\end{array}$ \\
\hline Adductor Longus & Pubic tubercle & $\begin{array}{l}\text { Medial lip of linea } \\
\text { aspera }\end{array}$ & $\begin{array}{l}\text { Adduct the hip; } \\
\text { Medially rotate the } \\
\text { hip }\end{array}$ & Obturator L2-4 \\
\hline Adductor Brevis & $\begin{array}{l}\text { Inferior ramus of } \\
\text { pubis }\end{array}$ & $\begin{array}{l}\text { Pectineal line and } \\
\text { medial lip of linea } \\
\text { aspera }\end{array}$ & $\begin{array}{l}\text { Adduct the hip; } \\
\text { Medially rotate the } \\
\text { hip }\end{array}$ & Obturator L2-4 \\
\hline
\end{tabular}

\section{Lumbopelvic Control (LPC)}

Similar to the anatomical structures of the LPHC, researchers cannot agree on a universal definition of LPC. ${ }^{4,5,31,35,41,45}$ The definition of LPC varies due to the differences in anatomical structures, what structures are being stabilized, and what muscular structures are being activated. ${ }^{31,45}$ For the purpose of this study, the following definition of LPC is used, "the ability of the neuromuscular system to control and protect the lumbar spine and pelvis from abnormal movements in all three planes (frontal, sagittal, and transverse) and maintain a neutral position". 4 
12-14, 31 This definition closely relates to the definition of balance, which will be discussed later. Basketball players move in multiple directions while running on the court while ball handling, boxing out, rebounding, jump shots, and defensive positions all while putting the lumbar spine and pelvis in abnormal positions.

The activation of the LPHC musculature provides stiffness/stabilization around the central mass of the trunk and pelvis, which contributes stability to the center of gravity of the body. ${ }^{14}$ A study conducted by Hodges and Richardson, ${ }^{55}$ used electromyography (EMG) to detect that the muscles of the LPHC activated every time before activation of the muscles in the extremities. ${ }^{5,35}$ The LPHC is also important for the distribution of forces. ${ }^{14,22,36}$ The LPHC has the largest amount of forces placed on it in the body, and when the LPHC is strong than the forces can be equally distributed to the extremities through the kinetic chain. ${ }^{14,21,22,36}$ The most relevant concept Kibler et al. ${ }^{14}$ discussed, is that the LPHC provides "proximal stability for distal mobility". 43, 35 This concept means, when the LPHC remains in a stable, neutral position, the upper and lower extremities gain more functional movement and acceleration of movements. ${ }^{4,14,}$ ${ }^{33,35}$ A study, conducted by King, ${ }^{53}$ and another study, conducted by Peterson, ${ }^{52}$ discussed the importance of postural exercises as posture dysfunctions can effect LPC in creating tissue stresses.' An example, discussed by Willson et al., ${ }^{35}$ described the knee and ankle movements being dependent on hip stabilization during jumping skills to preserve forward motion of acceleration over the center of gravity. This concept can be generalized to describe the LPHC remaining in a stable position in order to oppose internal and external perturbations. ${ }^{4}$

Instability of the LPHC can be caused by any deviation of the lumbar spine or pelvic girdle from the standard neutral position. ${ }^{13}$ The musculature of the LPHC is strengthened to avoid this instability and encourage the athlete to perform in a spine neutral manner. The 
transverse abdominis and multifidus are highly regarded as the two most important muscles for LPC. ${ }^{35}$ Exercise interventions for LPC are created with the basis of strengthening these two muscles. ${ }^{34}$ These individual muscles are also the basis for testing the athlete to quantify the LPC in existence.

Measurements of Lumbopelvic Control

Many different clinical tests were created to test LPC. These tests vary in validity and reliability for testing LPC due to different terminology used to define LPC, and also the disagreement of which muscle groups should be tested in order to properly measure LPC. When choosing which tests to use, researchers consider what muscles should be tested and what functional movements are to be evaluated. Biomechanical movement of the basketball player evaluated by the single leg raise test and abdominal fatigue test are very relevant for LPC in basketball players due to the motions of the single leg raise test being similar to motions in basketball and the abdominal fatigue test being able to test the strength of the LPHC muscle's stability.

The Level Belt Pro application was developed by Chaudhari and McKenzie. ${ }^{18}$ The application was created to measure anterior/posterior/lateral pelvic tilt and to determine an individual person's neutral pelvic tilt. Yedimenko et al. ${ }^{19}$ conducted a study on the inter-rater reliability and intra-rater reliability of the Level Belt Pro application used in conjunction with the single leg raise test for pelvic tilt measurements. The intra-rater reliability was shown to be excellent, but the inter-rater reliability was shown to be poor. This means the single leg raise test could be reliable in a study with only one rater, but would require additional training for studies that would have two or more raters. ${ }^{19}$ Chaudhari et al. ${ }^{4}$ conducted a study on professional baseball pitchers using the Level Belt Pro application, which he cocreated, and the single leg raise test to measure LPC. ${ }^{4}$ The single leg raise test is performed by placing 
the CoreX belt with iPod-based sensor (Level Belt Pro application) level on the hips lining up with the ASIS and PSIS. ${ }^{4}$ The athlete stands shoulder width feet apart then slowly raises one leg three inches off the ground and slowly lowers it back down. ${ }^{4}$ Chaudhari et al. ${ }^{4}$ conducted his research with only professional baseball pitchers, but this test can relate to basketball players as the motion of the single leg raise test is comparable to the form of a jump shot and lay-up. The SLRT was compared to 3D motion analysis for validation showing high sensitivity, specificity, and accuracy. ${ }^{4}$

Another test that can be used for LPC is the trunk flexor test, also called the flexor endurance test or the abdominal fatigue test (Table B2). The trunk flexor test tests the endurance of the LPHC musculature to see how long the musculature can maintain a spine neutral position by stabilizing around the spine. ${ }^{5}$ The relevance of this test is to measure the strength of the muscles to see if there is weakness; if there is weakness then the spine cannot be properly stabilized to internal or external perturbations. The test is performed by the athlete seated on a table with their hips and knees at a 90-degree angle with the upper body at 60-degrees in relation to the table. ${ }^{5,20-22}$ The feet are strapped to the table, and the athlete holds the 60 -degree angle for as long as possible. ${ }^{5,20-22}$ Test re-test reliability was found to have ICC scores of 0.95-0.98. Endurance times and ratios between tests are used as guidelines to interpret results. ${ }^{5,20-22}$

Other tests used for measuring LPC are the single leg squat test, Beiring-Sorensen backextension endurance test, side plank test, and abdominal drawing-in test (Table B2). The single leg squat test, Beiring-Sorensen back-extension endurance test, and side plank test all measure for lumbopelvic (core) endurance/strength more than LPC. ${ }^{5,20,21,33,35,36,40}$ The ICC for the Beiring-Sorensen back-extension endurance test is $0.97 .^{33}$ The ICC for the side plank test is 0.99. ${ }^{33}$ A study conducted by McGill et al. ${ }^{20}$ showed excellent reliability coefficients of .98 for the Beiring-Sorensen back-extension endurance and .99 for the side plank test on the left and 
right sides. The single leg squat test was shown to have inter-rater reliability with low to moderate agreement. ${ }^{33}$ The reliability was only tested with subject's dominant leg. ${ }^{33}$ The abdominal drawing-in test is used to measure lumbopelvic control by testing the stabilization of the transverse abdominis and multifidus when they activate. ${ }^{22}$ EMG activation was used to test for reliability. ${ }^{22}$

Table B2. Other measurements of Lumbopelvic control

Test $\underset{56}{\text { Single Leg Squat Test }}{ }^{20,31,33,35,}$ Beiring-Sorensen back-extension endurance test ${ }^{5,20,21,33.35,36,40}$

Side Plank Test $5,21,31,35,40$

Abdominal Drawing-in test ${ }^{13,22}$
Procedure

Standing on the test leg with the hip and knee in a neutral anatomical position.

Subject was positioned in prone lying position with pelvis at edge of the treatment table, with the pelvis and legs well stabilized; subject then crosses arms and assumes a horizontal position, subject then holds for as long as possible

Subjects lying on the table, on their side with legs extended. The top foot was placed in front of the lower foot on the mat for support. Subjects were instructed to support themselves lifting their hips of the mat to maintain a straight line over their full body length and supported themselves on the elbow and their feet. The uninvolved arm was held across the chest or simply rested on the lateral thigh of the upper extremity.

Subject lying in hook lying position, Biofeedback device placed under lumbar spine; subject performs drawing-in maneuver; subject then slowly extends the lower extremity by sliding heels down the table, Subject must maintain pressure during leg slide

\section{Balance and Proprioception}

Balance is very important to everyday activities such as walking, running, walking up stairs, and jumping to name a few. ${ }^{9,42}$ Balance is considered the ability to maintain the body's center of gravity over the base of support and results from neuromuscular actions in response to continuous visual, vestibular, and somatosensory feedback. ${ }^{6,9,11,37}$ As long as the disruptions to body do not cause disruptions to the center of gravity, the body will remain in balance. ${ }^{46}$ Balance 
is regulated in the body by the homeostasis process, which is the process where the internal environment is maintained and controlled within an organism despite the placement of external perturbations. ${ }^{47}$ Feedback is one of two control systems within the body that maintain the homeostasis of the human body, the other being feedforward. ${ }^{9,}{ }^{47}$ The feedback system is a response after sensory detection, while feedforward process is anticipated actions before sensory detection. $9,12,47,54$ The somatosensory, visual, and vestibular stimuli provide the information for these control systems. ${ }^{37,47}$ The somatosensory stimuli, also known as proprioception, refers to the detection of sensory stimuli. ${ }^{57}$ The visual stimuli refers to the stimuli received from the body's visual cues which is used to orientate the body within space. ${ }^{57}$ The vestibular stimuli refers to the information received through the ear canals and vestibules. ${ }^{57}$ The feedback control is considered more important to maintaining control as it provides the response to afferent information which consists of external perturbations. ${ }^{47}$ Mechanisms are the motor patterns the body performs to restore homeostasis. ${ }^{41,47}$ There are many processes within a mechanism including mechanoreceptor stimulation, neural transmission, integration of the signals by the central nervous system (CNS), transmission of an efferent signal, and force production. ${ }^{47}$ Each of these processes help in restoring homeostasis to the body when external perturbations are introduced. ${ }^{47}$ Stability occurs when the body remains unchanged even though disruptive forces are being applied. ${ }^{37,41,47}$ Neuromuscular control is the nervous system's control over the muscle activation during a particular task. ${ }^{37,47}$ In joint stability, neuromuscular control is important for the unconscious activation of the muscles in order to maintain the stability from being disturbed by external forces and movements. ${ }^{37,47}$

Proprioception, a function of the somatosensory system, is described as afferent information from the peripheral senses in order to maintain total posture, segmental posture, and 
muscle senses (posture, passive movement, active movement, and resistance to movement). ${ }^{9,} 11$, 37, 47, 57 Mechanoreceptors, also known as proprioceptors when transmitting proprioceptive information, are found in the muscle, tendon, ligament, and capsule structures in the body. ${ }^{47,57}$ Mechanoreceptors are used for transmitting the proprioceptive information through the afferent system in order to inform the central nervous system a response is needed. ${ }^{47,57}$ Ruffini receptors and Pacinian receptors can be found in the ligamentous and capsular tissues in order to adapt to dynamic movement. Two different mechanoreceptors can be found in the muscle tissue including Golgi tendon organs (GTOs) and muscle spindles. ${ }^{47,57}$ GTOs are responsible for sending feedback to the central nervous system in regard to increased muscle tension, mainly active muscle tension. ${ }^{47}{ }^{57}$ Muscle spindles send information to the central nervous system about muscle length and the rate the muscle length changes. ${ }^{47,57}$ These two mechanoreceptors work to send proprioceptive information of muscle action in order to control muscle activation. ${ }^{47,57}$ Balance is dependent on the postural control system, which consists of the sensory system, central nervous system, and musculo-skeletal system. ${ }^{58}$ The sensorimotor system consists of the sensory, motor, and central integration and processing components that maintain joint homeostasis during functional movements. ${ }^{47}$ These components help with maintaining functional joint stability along with static (bone, joint, and cartilage) and dynamic components (skeletal muscles). ${ }^{47}$ The central integration and processing components consists of the spinal cord, brain stem, and cerebral cortex. ${ }^{47,57}$ Two other areas that that help with motor control in the sensorimotor system are the cerebellum and basal ganglia, which help with regulating the motor commands. ${ }^{47}$ The central nervous system sends the signals from the brain to the muscle to react toward internal and external perturbations and return the body back to the center of gravity. ${ }^{47,57,}$ ${ }^{58}$ The spinal cord helps direct the motor responses and afferent and sensory information to 
appropriate destinations in order for the body to respond. ${ }^{47}$ The brain stem controls the postural equilibrium and automatic body movements. ${ }^{47}$ The brain stem can be divided into descending pathways or medial and lateral pathways. These pathways send responses to muscles with the lateral pathway controlling the distal extremity muscles and the medial pathway controlling the axial and proximal muscles. ${ }^{47}$ The cerebral cortex of the brain is in charge of controlling complex voluntary movements of the body. ${ }^{47}$

Balance can be divided into two separate divisions: static and dynamic. Dynamic balance is the ability to perform a task while maintaining a stable position. ${ }^{8,11}$ Static balance is the maintenance of a base of support with minimal to no movement. ${ }^{8}$ Dynamic balance has a greater relevance in athletics than static balance as it relates to stability during functional movement. ${ }^{8}$ Balance training is becoming a very popular trend for strength and conditioning. Struzik et al. ${ }^{46}$ stated that balance training can help improve postural control. A study conducted by Yaggie et al. ${ }^{11}$ showed balance training can improve a few sport-specific skills such as total sway and time on ball. This creates the basis for future research to see if increasing balance actually can improve specific skills in basketball such as accelerations/decelerations and changes of direction. ${ }^{9,46}$ Struzik et al. ${ }^{46}$ stated that basketball players exhibited a greater level of balance when compared to other sports due to the control over body and the ball simultaneously. Disequilibrium can hinder the performance of specific basketball skills such as the twisting of feet, jump shots, and offensive/defensive rebounds. ${ }^{9,}{ }^{46}$ Coordination abilities are an important fundamental aspect of sports and those abilities depend on movement control and regulation processes, which overall stated balance is important. ${ }^{8}$ Boccolini et al. ${ }^{6}$ stated that the sport of basketball forces the individual player into various situations of balance instability, such as accelerations/decelerations, changes in direction, penetrations into the defensive perimeter, 
boxing out, dribbling, and defense position recovery, while receiving physical contact from other players on the court. Filipa et al. ${ }^{17}$ concluded that "a neuromuscular training program that is focused on core stability, specifically the lower extremity strength, would affect the performance of an athlete in the Star Excursion Balance Test" since it assesses dynamic balance. ${ }^{6}$ Filipa et al. ${ }^{17}$ asserted there may be a significant relationship between lumbopelvic control and balance. Measurements of Balance

The Star Excursion Balance Test's (SEBT) purpose is to assess the dynamic balance ability of an athlete and/or healthy individual. ${ }^{23}$ The dynamic balance of an athlete is important as it correlates back to functional movement. Dynamic balance is measured in eight separate directions using single leg squat exercises which require full range of motion of all lower extremity joints, muscular strength, and proprioceptive/neuromuscular adjustments. ${ }^{23}$ The SEBT has shown to be very reliable when determining dynamic balance. ${ }^{23}$ The SEBT is the most reliable, quick method to measuring dynamic balance versus the gold standard which requires expensive computer machinery and time to complete. ${ }^{3}$ The traditional SEBT is performed by measuring eight separate directions 45 degrees from each other in a circle. The modified SEBT protocol is set up by first taping down measuring tapes in the anterior, posterolateral, and posteromedial directions.

Before conducting the SEBT, the researcher should collect height and leg length, Gribble et al. ${ }^{25}$ revealed no significant differences in the results between the right and left leg, but they did show differences in results between athletes with different lower leg limb lengths and height. There were no relations between foot type, hip rotation range of motion, or ankle range of motion, and excursion distance. ${ }^{25}$ Plisky et al. ${ }^{3}$ discussed the role of lower limb length and height, which can affect the results of the reach in the different directions. The recommendation to 
improve reliability is to have the athlete practice each direction four times. ${ }^{23,24}$ The measurements do not count if the athlete cannot maintain the single leg stance, the heel of the stance foot does not remain planted on the ground, or the athlete does not return to start position after each reach. ${ }^{17}$ Limitations to using the SEBT is that performing this test can be time intensive and can be variable interrater and intrarater reliability. ${ }^{23}$ Intrarater reliability was shown to more reliable than interrater reliability results. ${ }^{17,23,24}$ The protocol reliability will increase when rated by one researcher in the anterior, posterolateral, and posteromedial directions as these directions have been shown to have the best intrarater reliability. ${ }^{17,23,24}$ These directions are used as they have shown to be the most reliable in regard to basketball, as the three directions are the most travelled during activity. Dynamic balance is important in athletics, especially basketball. ${ }^{8}$ Further, the SEBT can be used in the basketball player to detect deficits in dynamic balance. Intervention Exercises for LPC

Training program exercises for lumbopelvic control are dependent upon the purpose of the study (i.e. stability, strength, or endurance), and what muscles are being targeted. Research shows that all lumbopelvic training programs should begin with contraction and static holds of musculature to improve muscular activation..$^{13,15-17,42,59}$ These contract and static hold exercises should focus on all musculature surrounding the lumbar spine and pelvis..$^{13,15-17,42,59}$ Early activation exercises should improve gradually with difficulty into quadruped and standing position. ${ }^{13,15-17,42,59}$ When the activation and holding exercises are improved, a stable base is created that will maintain the stabilization of the lumbopelvic-hip complex. ${ }^{15}$ Functional activities should be conducted while maintaining the contraction of the lumbopelvic-hip complex muscles. $^{15,16}$ 
Kahle et al. ${ }^{41}$ and Filipa et al. ${ }^{17}$ both looked at increasing Star Balance Excursion distances, which determines an increase in dynamic balance, by having subjects perform LPC training programs. Both showed improvements of reach distances and increases in dynamic balance, Exercises used in Filipa et al. ${ }^{17}$ consisted of hops and jumps in the all four directions, single leg holds progressing from stable to unstable surfaces, crunches, and back extension exercises. In the Kahle et al. study, ${ }^{42}$ bridge with leg lift, static abs, lower trunk rotation, planks, bicycles, full vertical crunches, bridges with marching, long arm crunches, bilateral leg lowering, and trunk rotation with weight were used. Mills et al. ${ }^{13}$ and Tse et al. ${ }^{59}$ both looked at how core stability training programs could improve performance. Mills et $\mathrm{al}^{13}$ used a ten-week training program, and Tse et al. ${ }^{59}$ used an eight-week training program. Both studies did not show any significant results in improving core. ${ }^{13}$ Mills et al. used leg lift and different types of bridge exercises compared to a group who performed only crunches and curl ups. The exercises Tse et al. ${ }^{59}$ used in their study were trunk stability exercises; specific exercises were not mentioned. Samson et al. ${ }^{60}$ evaluated tennis athletes indicating that it is important to perform core stabilization in order to improve performance using side bridge, dead bug, abdominal isometric contraction, and trunk twists. In theory, LPC is important for performance, and a LPC training program component should be incorporated into strength and conditioning training programs. Studies have shown though that there are no significant results of LPC training increasing performance. Previous studies have shown increases in other components of training, such as balance. Refer to Table B3 for more information.

$\underline{\text { Table B3. Intervention Exercises for Lumbopelvic Control }}$ 


\begin{tabular}{|c|c|c|c|c|}
\hline Samson et al. ${ }^{60}$ & $\begin{array}{l}\text { Increase of core } \\
\text { stabilization } \\
\text { program for tennis } \\
\text { players }\end{array}$ & $\mathrm{N} / \mathrm{A}$ & $\begin{array}{l}\text { Created an intervention based } \\
\text { on mastery of core } \\
\text { contraction, static holds and } \\
\text { slow movements in stable } \\
\text { environment, static holds in } \\
\text { unstable environment, } \\
\text { dynamic movement in } \\
\text { unstable environment, resisted } \\
\text { dynamic movement in } \\
\text { unstable environment }\end{array}$ & $\begin{array}{l}\text { Tennis needs a core } \\
\text { stabilization } \\
\text { program to improve } \\
\text { core for effective } \\
\text { performance }\end{array}$ \\
\hline Tse et al. ${ }^{59}$ & $\begin{array}{l}\text { Performance } \\
\text { increases in } \\
\text { college-aged } \\
\text { rowers }\end{array}$ & $\begin{array}{l}\text { Back extensor test, } \\
\text { abdominal fatigue } \\
\text { test, vertical jump, } \\
\text { standing broad } \\
\text { jump, 10-m shuttle } \\
\text { run, 40-m sprint, 2- } \\
\text { kg overhead } \\
\text { medicine ball } \\
\text { throw, 2,000-m } \\
\text { maximal rowing } \\
\text { ergometer test }\end{array}$ & $\begin{array}{l}\text { 8-week core stability program } \\
\text { starting with trunk activation } \\
\text { exercises gradually } \\
\text { progressing to more } \\
\text { functional exercises }\end{array}$ & $\begin{array}{l}\text { No significant } \\
\text { differences were } \\
\text { found for any of the } \\
\text { functional } \\
\text { performance tests }\end{array}$ \\
\hline Kahle et al. ${ }^{42}$ & $\begin{array}{l}\text { Compare effects of } \\
\text { a core stability } \\
\text { program on } \\
\text { dynamic balance }\end{array}$ & $\begin{array}{l}\text { Star Excursion } \\
\text { Balance Test }\end{array}$ & $\begin{array}{l}\text { Steadily progressing core } \\
\text { strengthening program three } \\
\text { times per week for } 6 \text { weeks }\end{array}$ & $\begin{array}{l}\text { Excursion distances } \\
\text { improved in core } \\
\text { stability exercise } \\
\text { group versus control } \\
\text { group }\end{array}$ \\
\hline Mills et al. ${ }^{13}$ & $\begin{array}{l}\text { Discover the } \\
\text { impact of a 10- } \\
\text { week training } \\
\text { regimen on } \\
\text { lumbopelvic } \\
\text { stability on } \\
\text { performance in } \\
\text { female athletes }\end{array}$ & $\begin{array}{l}\text { Pressure } \\
\text { Biofeedback Unit, } \\
\text { T-test, Sargent's, } \\
\text { Bass' test }\end{array}$ & $\begin{array}{l}10 \text { week program of } \\
\text { lumbopelvic stability } \\
\text { exercises that gradually } \\
\text { increased in functionality and } \\
\text { difficulty }\end{array}$ & $\begin{array}{l}\text { No significant } \\
\text { differences in } \\
\text { performance after } 10 \\
\text { week training } \\
\text { program }\end{array}$ \\
\hline Filipa et al. ${ }^{17}$ & $\begin{array}{l}\text { Determine if a } \\
\text { neuromuscular } \\
\text { training program } \\
\text { based on core and } \\
\text { lower body } \\
\text { exercises will } \\
\text { improve results of } \\
\text { SEBT on female } \\
\text { soccer players }\end{array}$ & $\begin{array}{l}\text { Star Excursion } \\
\text { Balance } \\
\text { test(SEBT) }\end{array}$ & $\begin{array}{l}\text { Eight week neuromuscular } \\
\text { training program consisting of } \\
\text { core and lower body strength } \\
\text { exercises, exercises were } \\
\text { performed biweekly }\end{array}$ & $\begin{array}{l}\text { Female soccer } \\
\text { players showed an } \\
\text { improvement of } \\
\text { SEBT distances after } \\
\text { completing } \\
\text { neuromuscular } \\
\text { training program }\end{array}$ \\
\hline
\end{tabular}

\section{Intervention Exercises for Balance}

Exercises that should be implemented in balance training programs should begin with stabilization of the spine and body on a stable ground. ${ }^{38}$ Difficulty in balance should be increased 
by introducing unstable surfaces to stance. ${ }^{38-40}$ Eyes can be closed to add difficulty to balance as it removes the visual stimuli that allows the central nervous system to stabilize the body. ${ }^{39,40}$ These exercises can be progressed by involving extra stimulus, such as throwing and receiving a ball, to help create more functional movement while maintaining balance. ${ }^{39,40}$

The majority of research conducted on balance training programs was conducted in order to see if there was a decrease in injuries, and if balance exercises were appropriate in rehabilitation protocols. Gioftsidou et al. ${ }^{39}$ conducted a study on soccer players to see if impaired balance caused more injuries, which was evident in the study. The exercises used were foot pressure on a Biodex platform, single leg stance with increasing unstable surfaces, and single limb stance on a mini trampoline. ${ }^{39}$ McGuine et al. ${ }^{28}$ and Cruz-Diaz et al. ${ }^{38}$ conducted studies on improving ankle injuries (ankle sprains and chronic ankle instability) by performing balance specific exercises such as single leg stance with eyes closed and open on a board while throwing, catching, dribbling on one leg, and double leg stance while rotating the balance board." These studies show performing balance exercises help with decreasing injuries in athletes which shows balance training programs causes improvements. Aggarwal et al., ${ }^{40}$ Cohen et al., ${ }^{61}$ Boccolini et al., ${ }^{6}$ and McKeon et al. ${ }^{29}$ conducted studies showing improvements in dynamic balance in athletes each using different exercises while randomizing subjects to groups. Aggarwal et al. ${ }^{39}$ uses specific walking exercises, single leg stance, and single leg stance on unstable surface. Cohen et al. ${ }^{61}$ used static relieve holds, heel raises, and single leg stance Boccolini et al. ${ }^{6}$ used the Swiss ball for an unstable surface and had the subjects perform kneeling holds, two handed chest pass, and single limb stance. McKeon et al. ${ }^{29}$ used single limb exercises with increasing levels of difficulty and hop to stabilization exercises. Following an error system advancement to the next level of difficulty, all four studies showed improvements in dynamic balance after 
completing balance training programs. These studies form a base to show that performing balance training programs can help improve the amount of dynamic balance an athlete has. Each study used different forms of measure to determine the amount of dynamic balance improvement gained following the intervention. Thus, a universal form of measurement should be created in order to determine if the results show to be consistent. Refer to Table B4 for more information. Table B4. Intervention Exercises for Balance

\begin{tabular}{|c|c|c|c|c|}
\hline Author & Purpose & Measured & Intervention & Results \\
\hline $\begin{array}{l}\text { Gioftsidou et } \\
\text { al. }{ }^{39}\end{array}$ & $\begin{array}{l}\text { Effects of a } \\
\text { soccer training } \\
\text { session on } \\
\text { balance and } \\
\text { whether } \\
\text { performing it } \\
\text { before or after } \\
\text { regular soccer } \\
\text { training session } \\
\text { is effective }\end{array}$ & $\begin{array}{l}\text { Balance boards; } \\
\text { Biodex Stability } \\
\text { System }\end{array}$ & $\begin{array}{l}\text { (1) A 3-min attempt to } \\
\text { move a cursor depicting the } \\
\text { position of the center of foot } \\
\text { pressure to a specific target } \\
\text { on a screen while standing } \\
\text { on the Biodex platform. (2) } \\
\text { A } 45 \text {-s attempt to maintain } \\
\text { single-limb stance on board } \\
\text { la. (3) A } 45 \text {-s attempt to } \\
\text { maintain single-limb stance } \\
\text { on board } 1 \text { b. (4) A } 45-\mathrm{s} \\
\text { attempt to maintain single- } \\
\text { limb stance on board } 2 . \text { ( } 5) \\
\text { A } 45 \text {-s attempt to maintain } \\
\text { single-limb stance on a mini } \\
\text { trampoline. In all tasks of } \\
\text { single-limb stance, subjects } \\
\text { were asked to kick back } \\
\text { with their nonstanding leg a } \\
\text { soccer ball thrown at them } \\
\text { by the experimenter. Both } \\
\text { legs were trained, } 15 \mathrm{~s} \text { apart. }\end{array}$ & $\begin{array}{l}\text { Impaired balance } \\
\text { can cause } \\
\text { injuries; } \\
\text { implications for } \\
\text { rehabilitation }\end{array}$ \\
\hline McGuine et al. ${ }^{28}$ & $\begin{array}{l}\text { Balance } \\
\text { program can } \\
\text { reduce risk of } \\
\text { ankle sprains in } \\
\text { high school } \\
\text { athletes }\end{array}$ & $\mathrm{N} / \mathrm{A}$ & $\begin{array}{l}\text { Five phase balance program } \\
\text { of balance exercises that } \\
\text { involved increasing harder } \\
\text { levels of surfaces; } 1 \text { ) } \\
\text { maintaining a single leg } \\
\text { stance on a flat surface with } \\
\text { eyes open and closed; (2) } \\
\text { performing functional sport } \\
\text { activities such as throwing, } \\
\text { catching, and dribbling on } 1\end{array}$ & $\begin{array}{l}\text { Balance training } \\
\text { program will } \\
\text { reduce ankle } \\
\text { sprains }\end{array}$ \\
\hline
\end{tabular}


leg; (3) maintaining doubleleg stance while rotating the balance board; (4)

maintaining a single-leg stance on the balance board with eyes open and

closed; and (5) performing

functional sport activities

while

in single-leg stance on the board

Aggarwal et al. $^{40}$

Cruz-Diaz et al. $^{38}$

Cohen et al. ${ }^{61}$
Comare efficacy of lumbar core stability training with balance training
Effectiveness of

6 week balance

training on athletes with chronic ankle instability

Systematic review of balance training program to increase performance of gymnasts

Boccolini et al. ${ }^{6} \quad$ Effectiveness of balance training to improve balance and vertical jump abilities of
Stark balance

test, Star

Excursion

Balance test, multiple single leg hopping stabilization
One group performed a core stabilization program consisting of exercises to enhance activation of local stabilizer muscles; Second group underwent a balance training program consisting of ankle strengthening exercises and functional balance exercises; third group was control

Cumberland

Ankle Instability Tool, Star Excursion Balance Test, Numeric Rating Scale N/A /A

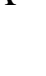

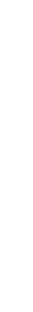

Libra board,
Bosco's
procedure jump
test

Balance exercises were performed on seven different surfaces: exercise mats, dynair, Bosu, mini tramp, foam roller, resistance band, and ankle disc

Created a balance program with emphasis of increasing ankle strengthening exercises

Subjects were separated into two groups: balance and isotonic, The balance group followed a training program with balance exercises with gradual difficulty, isotonic
Balance training and core training improve static and dynamic balance

Improvements of dynamic balance and chronic ankle instability were made

Creating a balance program will help improvements with gymnasts by increasing their dynamic balance

Balance training is effective for improving balance and vertical jump 
young

basketball

players

McKeon et al. ${ }^{29}$

Determine the

effect of a 4

week balance

training

program on

static and

dynamic

balance and

functional

outcomes on

those with

chronic ankle

instability group performed leg press

and leg extension exercises

Foot and Ankle Thirty-one young adults

Disability Index

(FADI); FADI

sport scales;

summary center

of pressures

excursion

measures; time-

to-boundary

measures;

modified Star

Excursion

Balance Test with chronic ankle

instability were randomly

assigned to a control group

and an intervention group.

The intervention group

performed a four week

balance training program

with exercises that

emphasize dynamic balance

in a single leg stance.
Balance training was effective in improving selfreported function, static postural control, and dynamic postural control.

\section{Summary}

Basketball is a popular sport and requires training different areas for improvement. LPC and balance training programs have become popular for coaches to implement during training. LPHC and LPC has many different definitions and use different terms to describe creating inconsistent literature. Many different clinical tests have been created to measure LPC; though to determine which test should be conducted, the researcher must decide on the structures (what muscles are being tested) and the function (stability, strength, or endurance) being measured. Balance is important in all aspects of movement and plays an important role in the basketball players control over the body and the ball simultaneously. The Star Excursion Balance Test is an appropriate measurement of dynamic balance, which is important to functional movement. Intervention programs for LPC and balance should be properly planned with exercises that will improve gradually in difficulty and be challenging to the athlete. The exercises should begin with first establishing stabilization then moving on to functional movement with stabilization. Balance 
exercises should be gradually increased in difficulty as well with different levels of unstable surfaces. 
APPENDIX C

ADDITIONAL METHODS

Table C1. Informed Consent

\begin{tabular}{ll} 
& \multicolumn{1}{c}{ Only Minimal Risk } \\
Principal Investigator & $\begin{array}{c}\text { Parental or Guardian Consent and HIPAA Form } \\
\text { Dr. Michelle A. Sandrey } \\
\text { College of Physical Activity and Sport Sciences }\end{array}$ \\
Department & $\begin{array}{l}\mathbf{1 6 1 0 2 9 9 0 6 9} \\
\text { Protocol Number } \\
\text { Study Title }\end{array}$ \\
program in High School Basketball Players \\
Co-Investigator(s) & Maggie Long, LAT, ATC
\end{tabular}

\section{Contact Persons}

In the event your child experiences any side effects or injury related to this research, you should contact Dr. Michelle A. Sandrey at (304) 293-0870. If you have any questions, concerns, or complaints about this research, you can contact Maggie Long, LAT, ATC at (219) 810-7935 or Dr. Michelle A. Sandrey at (304) 293-0870.

For information regarding your child's rights as a research subject, to discuss problems, concerns, or suggestions related to the research, to obtain information or offer input about the research, contact the Office of Research Integrity and Compliance (304) 293-7073.

In addition if you would like to discuss problems, concerns, have suggestions related to research, or would like to offer input about the research, contact the Office of Research Integrity and Compliance at 304-293-7073.

\section{Introduction}

Your child, __ has been asked to participate in this research study, which has been explained to you and your child by Maggie Long, LAT, ATC. This study is being conducted by Dr. Michelle A. Sandrey and Maggie Long, LAT, ATC at West Virginia University.This research is being conducted to fulfill the requirements for a masters thesis in Athletic Training in the Department of Sport Sciences at West Virginia University, under the supervision of Dr. Michelle A. Sandrey.

\section{Purpose(s) of the Study}

The purpose of this study is to improve your child's core stability and balance through a structured strength and conditioning program.

\section{Description of Procedures}

Before the commencement of the study, an orientation meeting will provide you the purpose and methods of this study. At this meeting, risk, discomforts, and confidentiality issues will be explained to you and your consent and demographic information will be obtained. Following this orientation, pre-test measures will commence.

This study involves performing three clinical tests that will measure core stability and balance, before and after performing a six-week strength and conditioning protocol. All tests and strength and conditioning protocols will 
be completed during open basketball gyms and basketball practices at Waynesburg Central High School unless asked otherwise.

The three clinical tests are the single leg raise test, abdominal fatigue test, and star excursion balance test. They will be performed as described below. They will be performed the week before the intervention begins and after the intervention as a post-test. All three tests should take about thirty minutes to complete.

Single Leg Raise Test

The single leg raise test is performed by placing the CoreX belt with iPod-based sensor (Level Belt Pro application) level on the hips lining up with the ASIS and PSIS. The athlete stands shoulder width feet apart then slowly raises one leg three inches off the ground and slowly lowers it back down.

Abdominal Fatigue Test

The test will be performed by the athlete seated on a table with the hips and knees at a 90-degree angle with the upper body at 60 -degrees in relation to the table. The feet will be strapped to the table, and the athlete will hold the 60-degree angle for as long as possible.

Star Excursion Balance Test

Athlete will begin in the starting position with both feet flat on the ground in the center of the grid with hands on both hips at all times. Instruct the athlete that they will keep stance foot directly on the ground without lifting their heel while reaching with the other leg in one direction. The athlete will try to touch the tape measure as far as possible with the most distal part of the foot once. After the touch, return to the starting position. Repeat in each direction. Once they have gone in each of the three direction stance legs will switch.

Following pretest procedures, you will undergo a six-week lumbopelvic control (core) and balance training program. The exercises will be those that have been recommended through the literature and practice that will improve strength, control, balance, and endurance. They are exercises that will include medicine balls, physioballs, and dumbbells and will be directed towards the lumbopelic-hip complex/core muscles and balance. The exercises will increase in difficulty from week to week. All exercises will be explained and shown by the co-principal investigator. The entire experiment will last 8 weeks with two weeks of pre-testing and post testing and 6 weeks of the training program that will take place during the first thirty minutes of open gym/practice.

\section{Risks and Discomforts}

There are no known or expected risks to your child from participating in this study. A feeling of discomfort and soreness from completing the exercises may be felt and that this will be no more than what is felt when learning a new exercise or skill. If an injury occurs during the study, Maggie Long, ATC will provide the necessary first aid and referral if necessary. I understand that the cost for the referral will be at my own expense.

\section{Alternatives}

Your child does not have to participate in this study.

\section{Benefits}

Your child may not receive any direct benefit from this study. The knowledge gained from this study may eventually benefit others by providing a "core" and balance training program for basketball. 


\section{Financial Considerations}

No payments will be made for participating in the study.

\section{Confidentiality}

Any information about your child that is obtained as a result of their participation in this research will be kept as confidential as legally possible.

Your child's research records and test results, just like hospital records, may be subpoenaed by court order or may be inspected by the study sponsor or federal regulatory authorities without your additional consent.

Audiotapes or videotapes will be kept locked up and will be destroyed as soon as possible after the research is finished.

In any publications that result from this research, neither your child's name nor any information from which your child might be identified will be published without your consent.

\section{HIPAA}

We know that information about your child and their health is private. We are dedicated to protecting the privacy of that information. Because of this promise, we must get your written authorization (permission) before we may use or disclose your child's protected health information or share it with others for research purposes.

You can decide to sign or not to sign this authorization section. However, if you choose not to sign this authorization, your child will not be able to take part in the research study. Whatever choice you make about this research study will not have an effect on your child's access to athletic training/medical care at Waynesburg Central High School.

\section{Persons/Organizations Providing the Information}

Basketball athlete on the Waynesburg Central High School basketball team/Waynesburg Central HS injury reports

\section{Persons/Organizations Receiving the Information}

- $\quad$ Health care providers who provide services to you as part of this research study: WVU, Michelle A. Sandrey, PhD, ATC, Maggie Long, ATC. The research site(s) carrying out this study includes WVU..

- The United State Department of Health and Human Services (which includes the National Institutes of Health (NIH), Food and Drug Administration (FDA)) and other groups that have the right to use the information as required by law.

- $\quad$ Foreign Regulatory Agencies

- $\quad$ The members and staff of any Institutional Review Board (IRB) that oversees this research study.

- West Virginia University Office of Research Integrity and Compliance and Office of Sponsored Programs.

- West Virginia University Clinical Trials Research Unit.

\section{The Following Information Will Be Used}


Information from your child's existing medical records and new information about them that is created or collected during the study such as: history and physicals, demographic data, and study forms.

\section{The Information is Being Disclosed for the Following Reasons}

- $\quad$ Review of your child's data for quality assurance purposes

- $\quad$ Publication of study results (without identifying your child)

- $\quad$ Other research purposes such as reviewing the safety or effectiveness of the study training program; conducting performance reviews of the training program; evaluating other products or therapies for patients; developing a better understanding of core and balance training programs; improving the design of future clinical trials

\section{You May Cancel this Authorization at Any Time by Writing to the Principal Investigator} Michelle A. Sandrey (304) 293-0870 msandrey@mail.wvu.edu If you cancel this authorization, any information that was collected already for this study cannot be withdrawn. Once information is disclosed, according to this authorization, the recipient may redisclose it and then the information may no longer be protected by federal regulations.

You have a right to see and make copies of your child's medical records. You will not be able to see or copy your child's records related to the study until the co-PI has completed all work related to the study. At that time you may ask to see the study files related to your child's participation in the study and have the co-PI correct any information about your child that is wrong.

This authorization will expire at the end of the study unless you cancel it before that.

\section{Voluntary Participation}

Refusal to participate or withdrawal will not affect your child's future care at Waynesburg Central HS, and will involve no penalty to you.

\section{Signatures}

Upon signing this consent, you will receive a copy.

I willing consent to allow my child to participate in this research.

Signature of Parent or Guardian

\begin{tabular}{lll}
\hline Printed Name & Date & Time
\end{tabular}

Signature of Investigator or Co-Investigator

\begin{tabular}{lll}
\hline Printed Name & Date & Time
\end{tabular}


Table C2. Informed Assent

\begin{tabular}{|c|c|}
\hline \multicolumn{2}{|r|}{ Assent Form } \\
\hline Principal Investigator & Dr. Michelle A Sandrey \\
\hline Department & College of Physical Activity and Sport Sciences \\
\hline Protocol Number & 1610299069 \\
\hline Study Title & $\begin{array}{l}\text { The Effects of a Six Week Lumbopelvic Control and Balance Training Program in } \\
\text { High School Basketball Players }\end{array}$ \\
\hline Co-Investigator(s) & Maggie Long, LAT, ATC \\
\hline
\end{tabular}

\section{Contact Persons}

If you are hurt from being in this research, you should contact Dr. Michelle Sandrey at (304) 293-0870. If you have any questions, concerns, or complaints about this research, you can contact Maggie Long, LAT, ATC at (219) 810-7935 or Dr. Michelle A. Sandrey at (304) 293-0870.

For information regarding your rights as a person in research or to talk about the research, call the Office of Research Integrity \& Compliance at (304) 293-7073.

\section{Introduction}

You, , have been asked to participate in this research study, which has been explained to you by Maggie Long, LAT, ATC.

\section{Purpose(s) of the Study}

The purpose of this study is to improve your core stability and balance through a structured strength and conditioning program.

\section{Description of Procedures}

Before the commencement of the study, an orientation meeting will provide you the purpose and methods of this study. At this meeting, risk, discomforts, and confidentiality issues will be explained to you and your consent and demographic information will be obtained. Following this orientation, pretest measures will commence.

This study involves performing three clinical tests, that will measure core stability and balance, before and after performing a six-week strength and conditioning protocol. All tests and strength and conditioning protocols will be completed during open basketball gyms and basketball practices at Waynesburg Central High School unless asked otherwise. The entire experiment will last 8 weeks with two weeks of pre-testing and post testing and 6 weeks of the training program.

The three clinical tests are the single leg raise test, abdominal fatigue test, and star excursion balance test. They will be performed as described below.

Single Leg Raise Test

The single leg raise test is performed by placing the CoreX belt with iPod-based sensor (Level Belt Pro application) level on the hips lining up with the ASIS and PSIS. The athlete stands shoulder width feet 
apart then slowly raises one leg three inches off the ground and slowly lowers it back down.

\section{Abdominal Fatigue Test}

The test will be performed by the athlete seated on a table with the hips and knees at a 90-degree angle with the upper body at 60-degrees in relation to the table. The feet will be strapped to the table, and the athlete holds the 60-degree angle for as long as possible.

Star Excursion Balance Test

Athlete will begin in the starting position with both feet flat on the ground in the center of the grid with hands on both hips at all times. Instruct athlete that they will keep stance foot directly on the ground without lifting their heel while reaching with the other leg in one direction. The athlete will try to touch the tape measure as far as possible with the most distal part of the foot once. After the touch, return to the starting position. Repeat in each direction. Once they have gone in each direction have them switch stance legs.

Following pretest procedures, you will undergo a six-week lumbopelvic control and balance training program. The exercises will be those that have been recommended through the literature and practice that will improve strength, control, balance, and endurance. They are exercises that will include medicine balls, physioballs, and dumbbells and will be directed towards the lumbopelvic-hip complex (core). The exercises will increase in difficulty from week to week. All exercises will be explained and shown by the principal investigator.

\section{Discomforts}

There are no known or expected risks to you from participating in this study. A feeling of discomfort and soreness form completing the exercises may be felt and that this will be no more than what is felt when learning a new exercise or skill. If an injury occurs during the study, Maggie Long, ATC will provide the necessary first aid and referral if necessary. I understand that the cost for the referral will be at my own expense

\section{Benefits}

This study may not help you, but what they learn from the study may help other people by providing a "core" and balance training program for basketball.

\section{Confidentiality}

We promise that anything we learn about you in this study will be kept as secret as possible.

\section{Voluntary Participation}

You do not have to do this. No one will be mad at you if you refuse to do this or if you decide to quit. You have been allowed to ask questions about the research, and all of your questions were answered.

I willingly agree to be in this research. 


\section{Signatures}

Signature of Subject

Printed Name

Date

Time

The minor has had the opportunity to have questions addressed. The minor willingly agrees to be in the study.

Signature of Investigator or Co-Investigator

Printed Name

Date

Time 
Table C3. Subject Demographics

Subject Number:

Age:

Height:

Weight:

Injury History

* Injuries are considered ones that were evaluated by an athletic trainer or medical doctor.

1. Have you had an upper extremity injury (shoulder, neck, upper back, arm) within the past six months? Yes/No If yes, Please explain:

2. Have you ever had a lower extremity injury (lower back, hips, legs) within the past six months? Yes/No If yes, Please explain:

3. Have you had a head injury within the past six months? Yes/No

If yes, please explain:

4. Have you had any neurological disorders (i.e. seizures, strokes) within the past six months? Yes/No If yes, please explain:

5. Have you had any vestibular disorders (problem with balance related to the ear) within the past six months? Yes/No

If yes, please explain:

6. Have you had visual (eyes) disorders within the past months? Yes/No

If yes, please explain:

7. Are you currently involved in any of the following physical activities? Circle all that apply

Weight Training

Cardiovascular Training

Pilates

Yoga

Other

If yes, please explain what physical activity you are involved in and how often you are involved in each activity:

8. Are you taken any medications that affect performance or balance? Yes/No

If yes, please explain:

9. Are you currently in a training program for balance? Yes/No

If yes, please explain:

10. Are you currently in a core stability program? Yes/No

If yes, please explain:

11. Are you currently in a training program to improve performance? Yes/No If yes, please explain:

12. Have you had any surgeries on either hips, shoulders, or your abdomen in the past year? Yes/No If yes, please explain: 
Table C4. Data Collection

Pre-Test

Subject Number

Date

Lumbopelvic Control

Level Belt Pro

Trial One

Trial Two

Average

Anterior/Posterior tilt- Left Leg

Anterior/Posterior tilt- Right Leg

Abdominal Fatigue Test

Trial One

Trial Two

Trial 3

Average

Time Held

Star Excursion Balance Test

Trial One

Trial Two

Trial 3

Average

Left Leg

Anterior

Posteromedial

Posterolateral

Right Leg

Anterior

Posteromedial

Posterolateral 
Post-Test

Subject Number

Date

Lumbopelvic Control

Level Belt Pro

Anterior/Posterior tilt- Left Leg

Anterior/Posterior tilt- Right Leg

Abdominal Fatigue Test

Time Held

Star Excursion Balance Test

Left Leg

Anterior

Posteromedial

Posterolateral

Right Leg

Anterior

Posteromedial

Posterolateral
Trial One

$-$

Trial One

Trial Two

Trial 3

Average

Trial One

Trial Two

Trial 3

Average

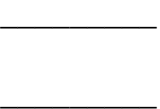

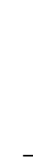

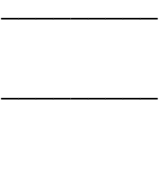

Trial Two

Average

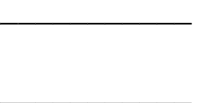

\section{Average}
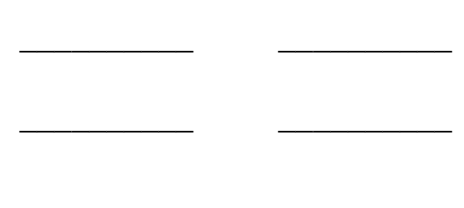
Table C5. Pre-test/Post Test

Single Leg Raise Test ${ }^{4,18}$
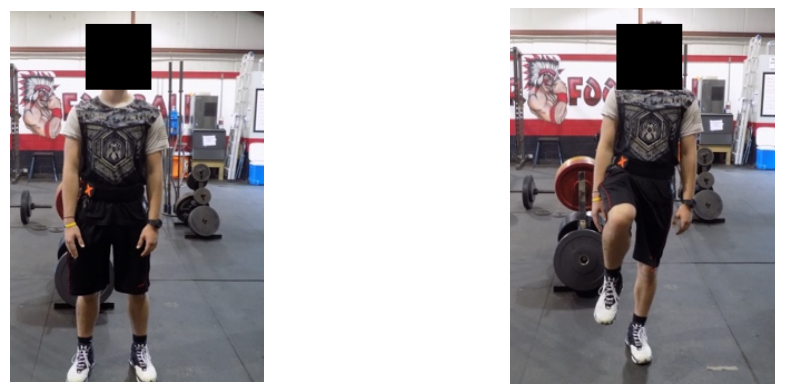

1. Place CoreX belt lined up with both ASIS and PSIS

2. Start with feet shoulder width apart and weight evenly distributed

3. Slowly lift one foot up as though you would step up onto a curb and hover approximately 3" above the floor

4. Hold single leg position for approximately 2 seconds

5. Record largest of two numbers repeated by Level Belt Pro App

6. Return to start position

7. Take belt off then and immediately replace it to realign with ASIS and PSIS

8. Repeat test. Record measure then average the two

9. Repeat on other leg

Abdominal Fatigue Test ${ }^{20}$
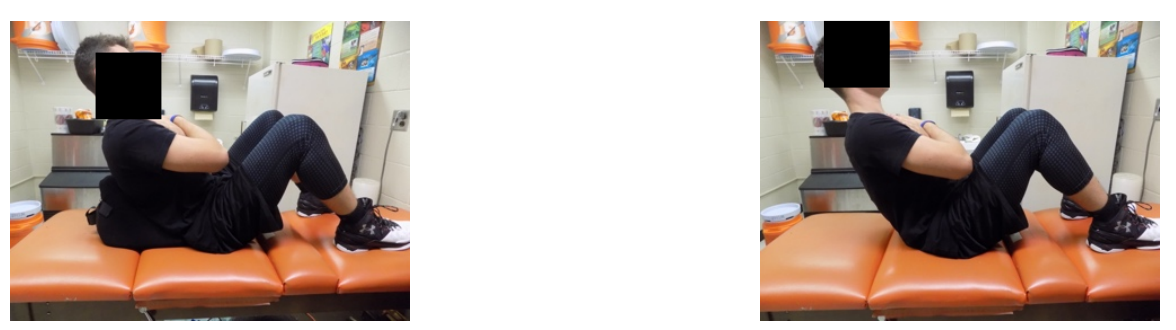

1. Athlete is seated on a table with the hips and knees at a 90-degree angle with the upper body at 60 -degrees in relation to the table

2. The feet are strapped to the table, and the athlete holds the 60-degree angle for as long as possible. 
3. A stopwatch is used to record the hold time

Star Excursion Balance Test ${ }^{23-25}$

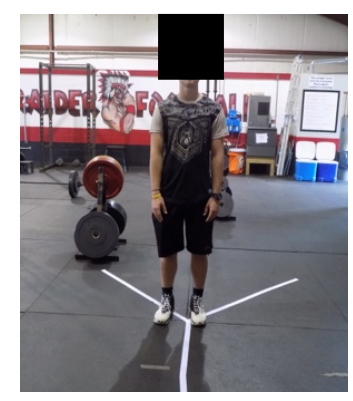

Beginning Stance

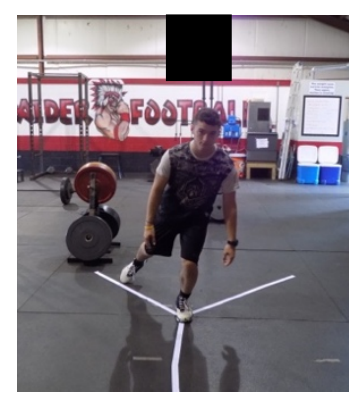

Posterolateral direction

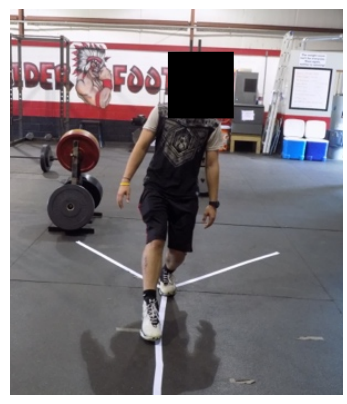

Anterior Direction

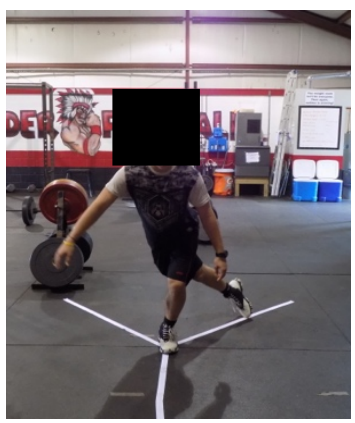

Posteromedial direction

1. Athlete will begin in the starting position with both feet flat on the ground in the center of the grid with hands on both hips at all times.

2. Instruct athlete that they will keep stance foot directly on the ground without lifting the heel while reaching with the other leg in one direction.

3. The athlete will try to touch the tape measure as far as possible with the most distal part of the foot once.

4. After the touch, return to the starting position.

5. Repeat in each direction

6. Once they have gone in each direction have them switch stance legs after a five minute rest period 
Table C6. Lumbopelvic Control/Balance Training Intervention Program 14-16, 26, 50

Week

1

2

3

4

Exercise
Supine Abdominal Hollowing
Supine Abdominal Bracing
Prone Bridge
Supine Bridge
Curl Up
Superman
Cat/Camel

Supine Bent Knee Raises

Side Bridge (Each Side)

Supine Single Leg Bridges

Standing Abdominal Hollowing

Standing Abdominal Bracing

Aqua Man

Cat/Camel with Eyes Closed

Prone Plank

Bird Dog

Glut Side Lying Snaps (Each Side)

Bridge on Physioball

Push Through

Cross Over Crunch

Single Leg Lift (Each Side)

Medicine Ball Sit Up

Side Plank

Dead Bug

Bicycles

March Ups on Physioball

Apollo Creed

\begin{tabular}{|c|c|c|}
\hline Sets & Repetitions & Weight/Resistance \\
\hline 3 sets & $30 \mathrm{sec}$ & N/A \\
\hline 3 sets & $30 \mathrm{sec}$ & $\mathrm{N} / \mathrm{A}$ \\
\hline 3 sets & $30 \mathrm{sec}$ & N/A \\
\hline 3 sets & $30 \mathrm{sec}$ & N/A \\
\hline 3 sets & 15 reps & N/A \\
\hline 3 sets & $30 \mathrm{sec}$. & N/A \\
\hline 3 sets & $15 \mathrm{sec}$. each position & $\mathrm{N} / \mathrm{A}$ \\
\hline 3 sets & 15 reps & $\mathrm{N} / \mathrm{A}$ \\
\hline 3 sets & $30 \mathrm{sec}$. & $\mathrm{N} / \mathrm{A}$ \\
\hline 3 sets & $30 \mathrm{sec}$. & N/A \\
\hline 3 sets & $30 \mathrm{sec}$. & N/A \\
\hline 3 sets & $30 \mathrm{sec}$. & N/A \\
\hline 3 sets & 15 reps & N/A \\
\hline 3 sets & $15 \mathrm{sec}$. each position & N/A \\
\hline 3 sets & $30 \mathrm{sec}$. & $\mathrm{N} / \mathrm{A}$ \\
\hline 3 sets & 15 reps & N/A \\
\hline 3 sets & 15 reps & N/A \\
\hline 3 sets & $30 \mathrm{sec}$. & $\mathrm{N} / \mathrm{A}$ \\
\hline 3 sets & 15 reps & N/A \\
\hline 3 sets & 15 reps & N/A \\
\hline 3 sets & 15 reps & N/A \\
\hline 3 sets & 15 reps & $5 \mathrm{lbs}$ \\
\hline 3 sets & $30 \mathrm{sec}$. & $\mathrm{N} / \mathrm{A}$ \\
\hline 3 sets & 15 reps & $\mathrm{N} / \mathrm{A}$ \\
\hline 3 sets & 15 reps & N/A \\
\hline 3 sets & 15 reps & N/A \\
\hline 3 sets & 15 reps & N/A \\
\hline
\end{tabular}


Medicine Ball Floor Twist

Double Leg Lift

Swimming on Knees
3 sets

3 sets

3 sets

3 sets

3 sets

3 sets

3 sets

3 sets

3 sets

3 sets
15 reps

15 reps

15 reps

15 reps

15 reps

15 reps

15 reps

15 reps

15 reps

15 reps

15 reps

15 reps

15 reps

15 reps

15 reps

15 reps

15 reps
$5 \mathrm{lbs}$

N/A

N/A

$5 \mathrm{lbs}$

$5 \mathrm{lbs}$

N/A

N/A

N/A

$5 \mathrm{lbs}$

$10 \mathrm{lbs}$

$5 \mathrm{lbs}$

N/A

$5 \mathrm{lbs}$

$10 \mathrm{lbs}$

N/A

$5 \mathrm{lbs}$

$5 \mathrm{lbs}$ 
Table C7. Lumbopelvic Control Intervention Phase 1 Exercises ${ }^{14-16,26,50}$

Supine Abdominal Hollowing

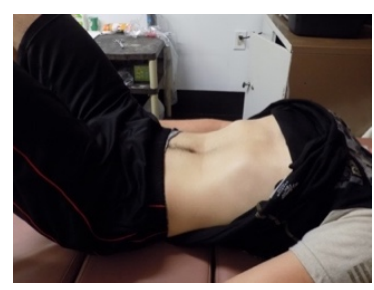

1. Draw in the abdomen; "a string pulling the belly button toward the spine"

Supine Abdominal Bracing

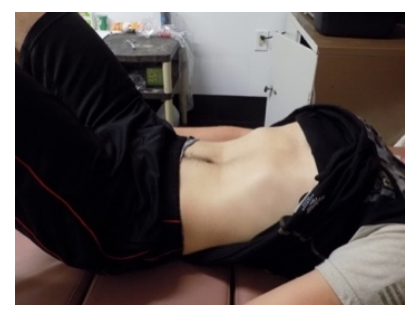

1. Widening the trunk; Not pulling in or pushing out

Prone Bridge
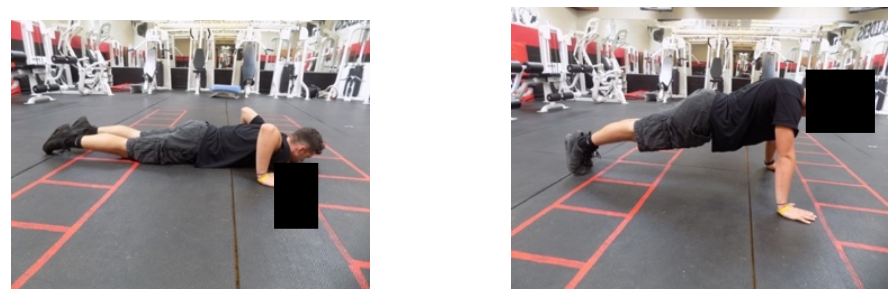

1. Assume a start push up position" Hands will be shoulder width apart; Draw core in towards spine holding a parallel position with the floor. 
Supine Bridge
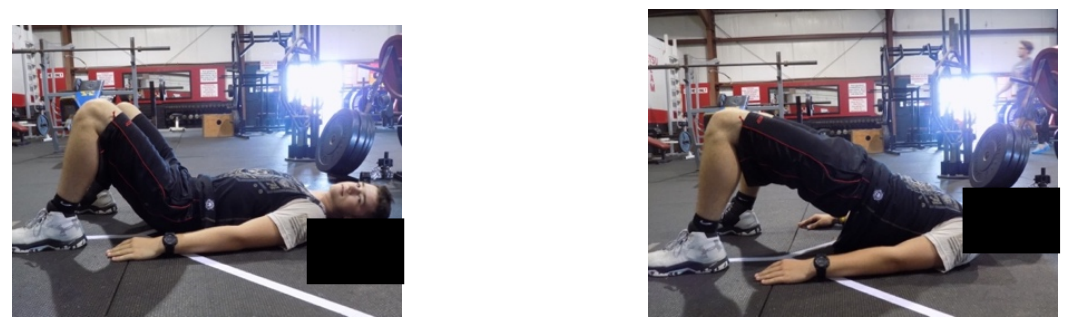

1. Lay supine (on your back); Arms stay lying on sides

2. Push hips up toward the sky; Drawing core in to spine and holding

Curl Up
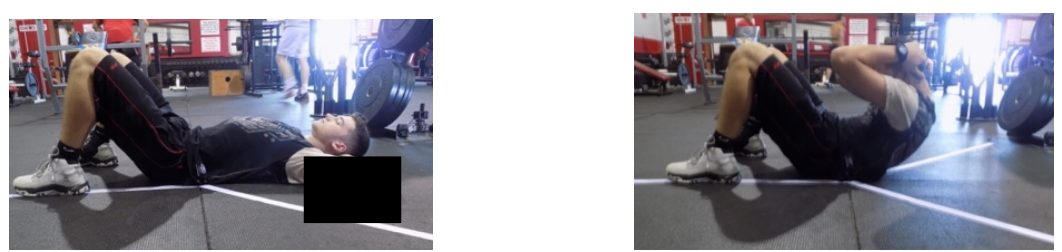

1. Lay supine (on your back); Arms behind head

2. Draw core in while bringing nose to knees

Superman
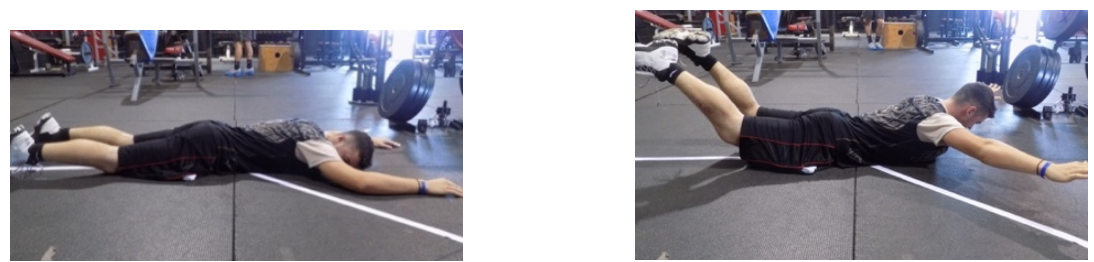

1. Lay prone (on stomach); Legs straight; Arms straight above head

2. Draw core in; Lifts both arms and legs up and hold 
Cat/Camel
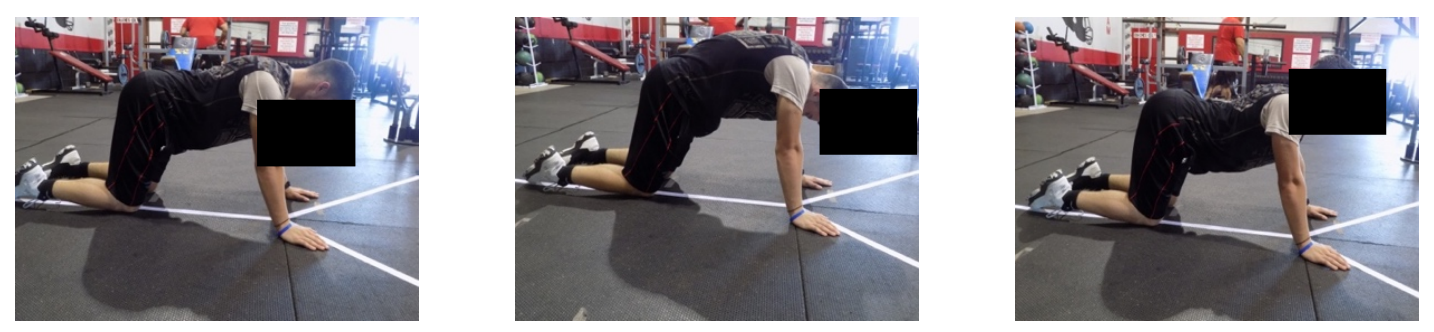

1. Begin in quadruped position (on hands and knees) with back straight

2. Draw in core, rounding back, tucking chin to chest

3. Draw spine to the ground, creating a bowl with the back, head up, chin out

Table C8. Lumbopelvic Control Intervention Phase 2 Exercises

Supine Bent Knee Raises
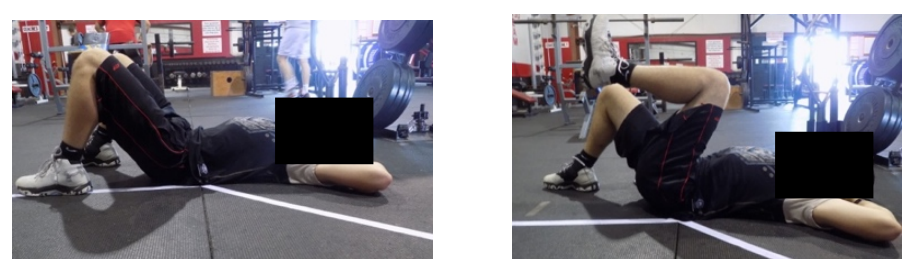

1. Lay supine (on your back); Arms behind head

2. Flex hip bringing knee to chest while keeping knee at 90 degrees

3. Repeat with other leg.

Side Bridge (Each Side)

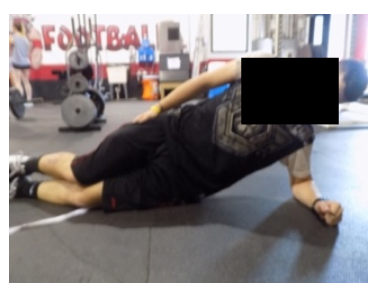

1. Lay on your side; Knees bent; Leaning on forearm

2. Lift hips off the ground; Draw core in 
Supine Single Leg Bridges
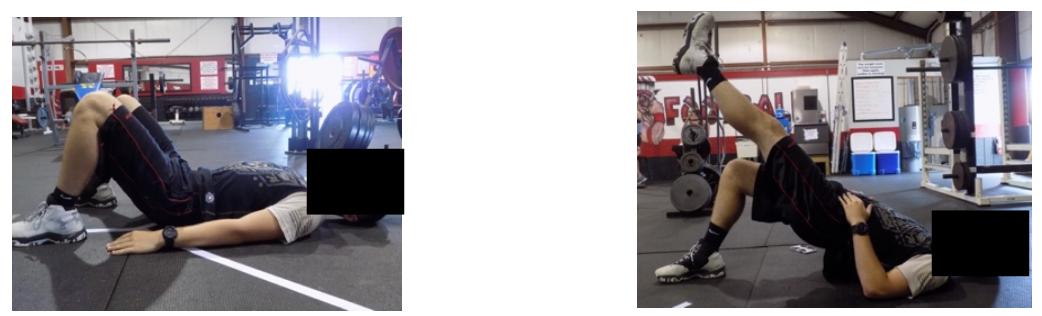

1. Lay supine (on your back); Arms stay lying on sides

2. Push hips up toward the sky; Drawing core in to spine and holding

3. Lift leg in line with core

4. Repeat on other side

Standing Abdominal Hollowing while dribbling

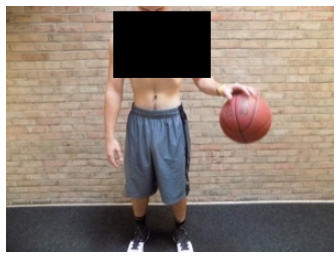

1. While standing, draw in the abdomen; "a string pulling the belly button toward the spine"

2. Standing Abdominal Bracing while dribbling

3. While standing, widening the trunk; Not pulling in or pushing out

Aqua Man
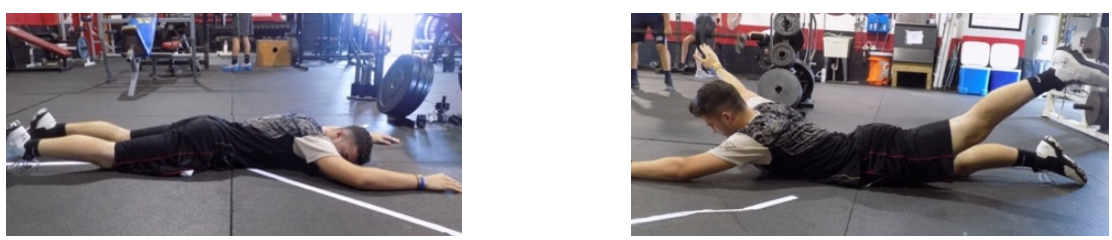

1. Lay prone (on stomach); Legs straight; Arms straight above head

2. Draw core in; Lifts alternate arm and leg up and hold

3. Repeat with opposite arm/leg 
Cat/Camel w/ Eyes Closed
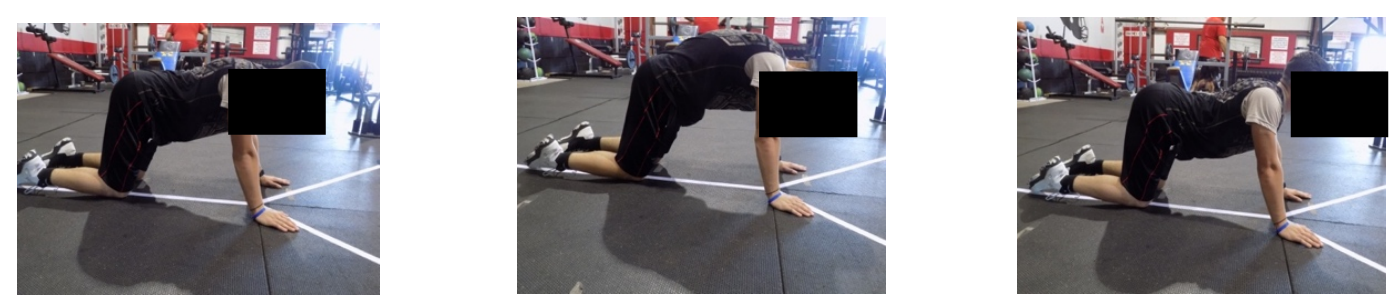

1. All performed with eyes closed

2. Begin in quadruped position (on hands and knees) with back straight

3. Draw in core, rounding back, tucking chin to chest

4. Draw spine to the ground, creating a bowl with the back, head up, chin out

Table C9. Lumbopelvic Control Intervention Phase 3 Exercises

Prone Plank

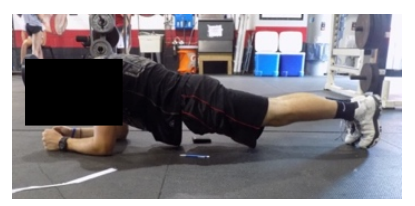

1. Begin laying prone (on stomach) on the ground

2. Rise up on elbow and toes

3. Draw core in to spine; hold body straight parallel to the ground

Bird Dog
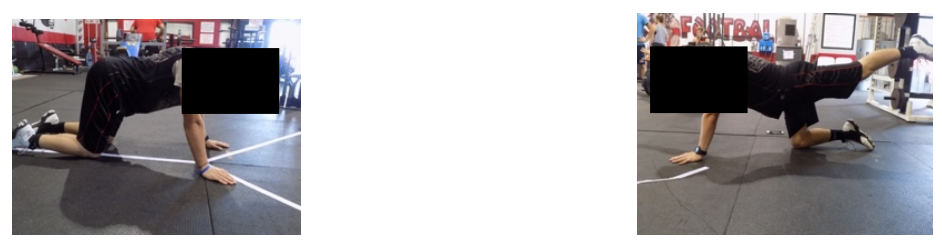

1. Begin in quadruped position (on hands and knees) with back straight

2. Raise opposite arm and leg to be parallel with the trunk

3. Lower to start position; repeat with other arm and leg 
Glut Side Lying Snaps
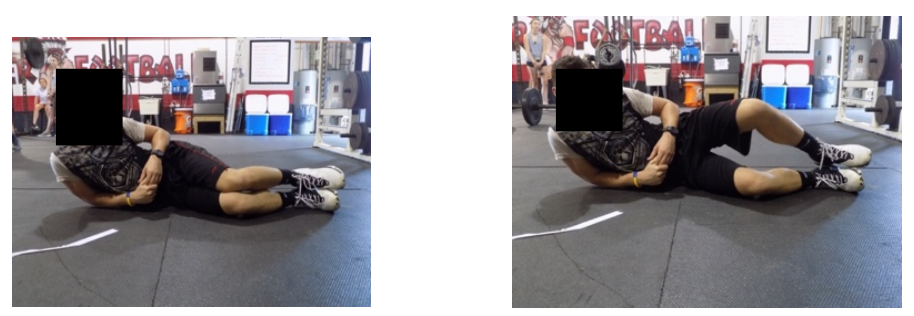

1. Lay on side with knees bent 45 degrees

2. Open legs with top foot rolling on bottom foot

3. Open as wide as possible then close legs back to start position

Bridge on Physioball
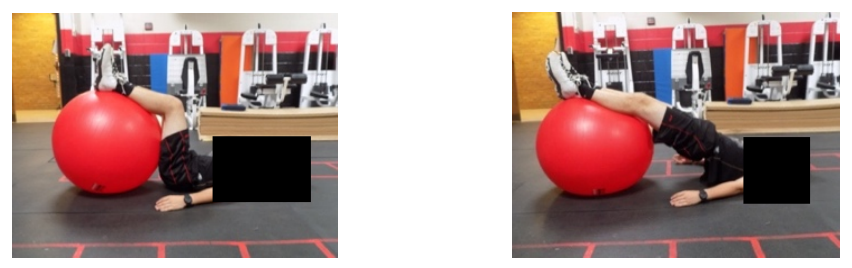

1. Lay supine (on your back); Arms stay lying on sides; Feet on top of physioball

2. Push hips up toward the sky; Drawing core in to spine and holding

Push Through
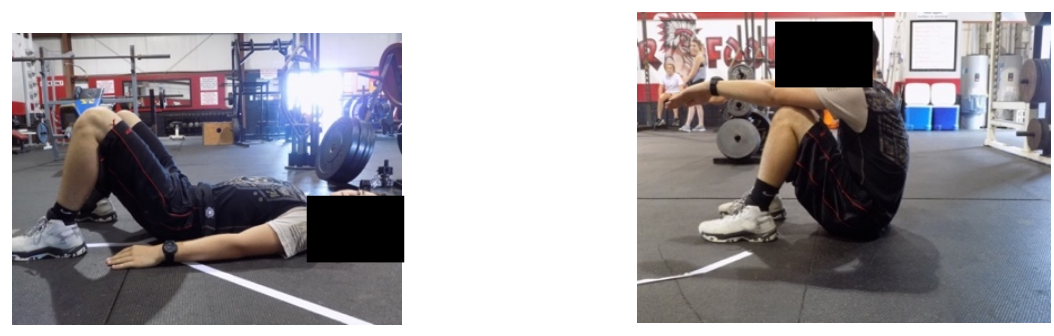

1. Lay supine (on your back); Arms stay lying on sides

2. Sit all the way up without feet lifting off ground; Push hands past knees

3. Lay back down to start position 
Cross Over Crunch
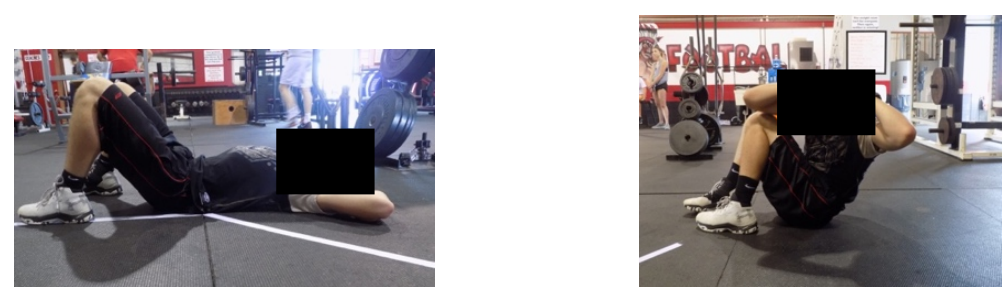

1. Lay supine (on your back); Arms behind head

2. Sit up with feet stationary on the ground

3. Touch elbow to knee twisting body to one side

4. Repeat on other side

Single Leg Lift
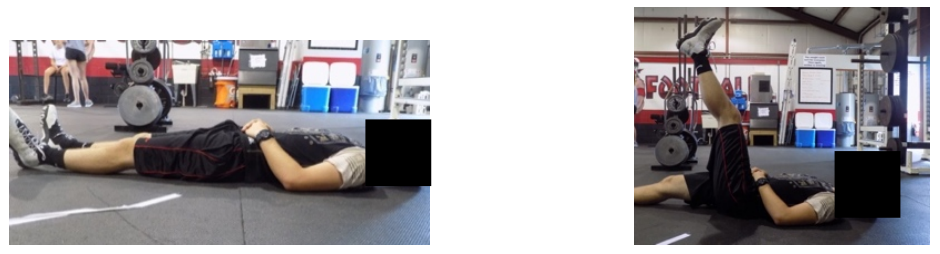

1. Lay supine (on back); legs straight; arms at sides

2. Lift one leg up to 90 degrees hip flexion; draw core in to spine

3. Repeat with other leg

Medicine Ball Sit Up
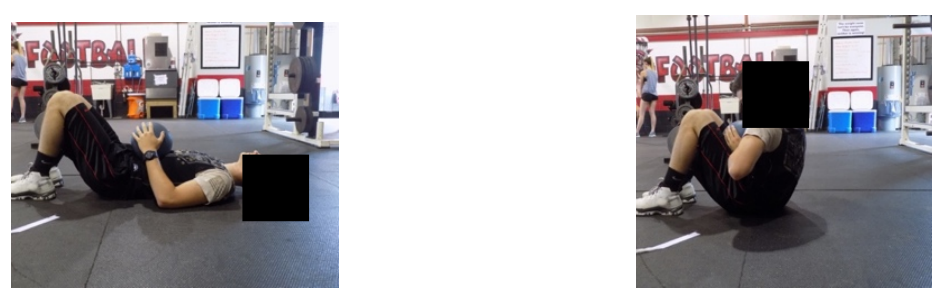

1. Lay supine (on your back); Arms stay lying on sides; Holding $5 \mathrm{lb}$ medicine ball

2. Sit all the way up without feet lifting off ground; Holding medicine ball

3. Lay back down to start position 
Table C10. Lumbopelvic Control Intervention Phase 4 Exercises

Side Plank

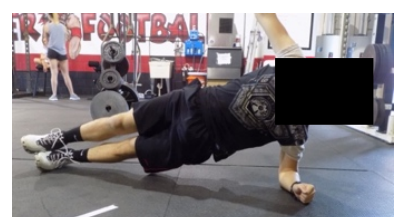

1. Lay on your side; Knees straight; Leaning on forearm

2. Lift hips off the ground; Draw core in

Dead Bug
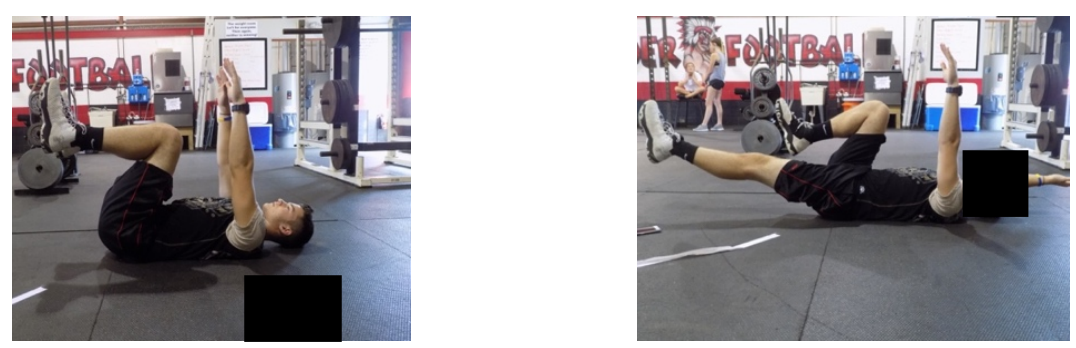

1. Begin in supine position (on back) with back straight; Hips and knees flexed to 90 degrees

2. Lower opposite arm and leg to be parallel with the trunk

3. Raise to start position

4. Repeat with other arm and leg

Bicycles
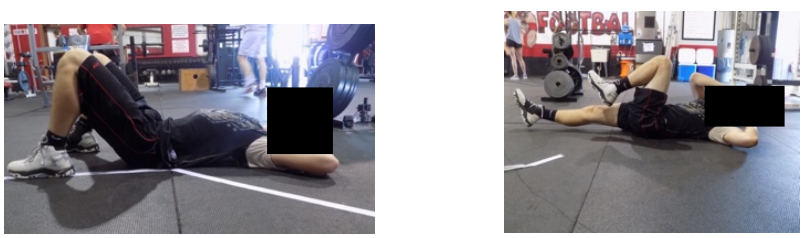

1. Lay supine (on your back); Arms behind head

2. Lift both legs 6 inches off ground

3. Bend knee and bring to chest

4. Touch elbow to knee twisting body to one side; repeat on other side 
March Ups on Physioball
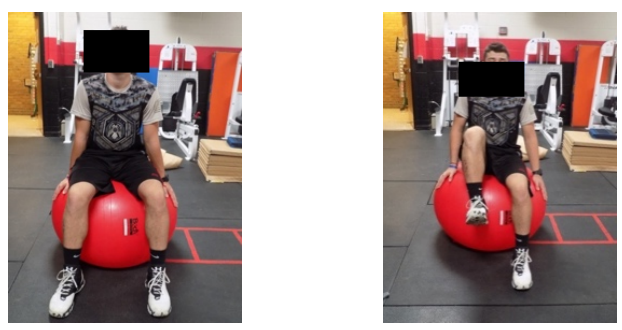

1. Begin sitting on physioball; feet shoulder width apart; knees parallel to ankles; core drawn in to spine

2. Raise knee as high as possible without compromising the core or moving off balanced on physioball

3. Repeat on other side

Apollo Creed
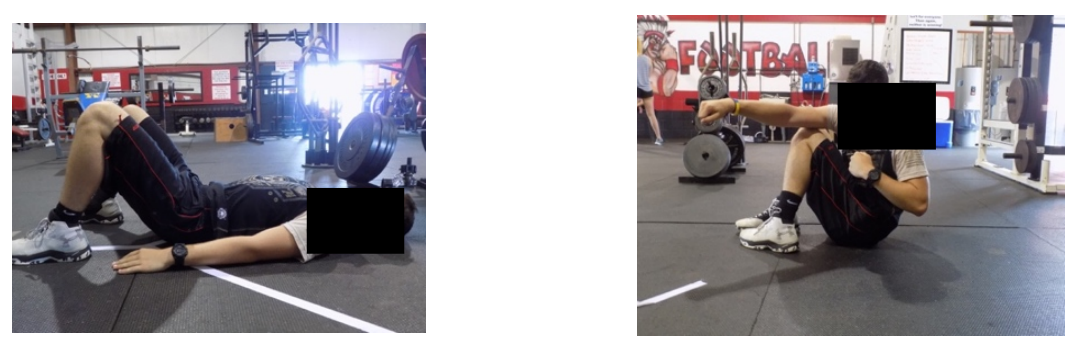

1. Lay supine (on your back); Arms stay lying on sides

2. Sit all the way up without feet lifting off ground; Punch across the body

3. Lay back down to start position; Repeat with opposite arm

Medicine Ball Floor Twists
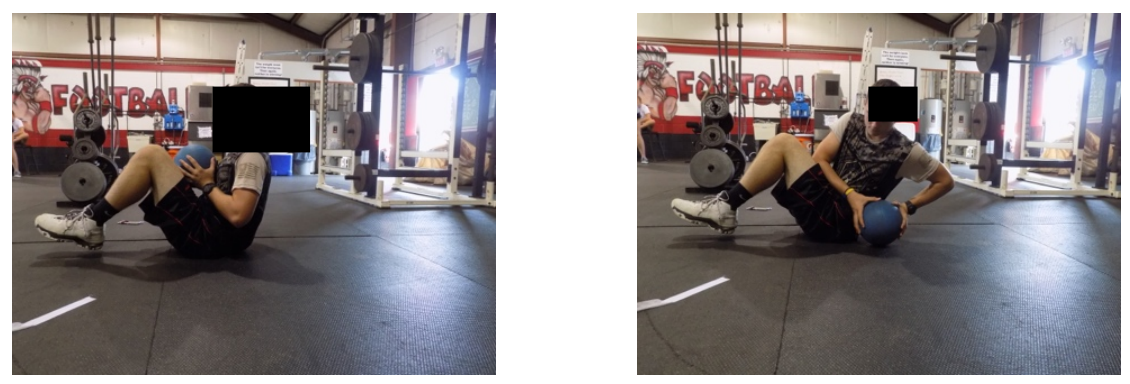

1. Begin with knees bent; hold medicine ball at chest level 
2. Raise feet six inches off ground; Draw core in to spine

3. Rotate body to each side touching the medicine ball to the floor while maintaining the core

Double Leg Lift
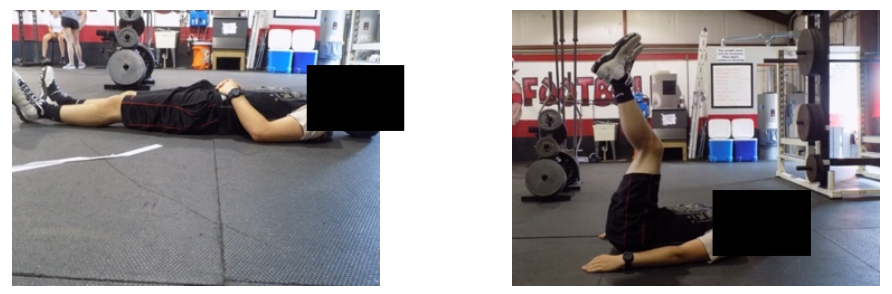

1. Lay supine (on back); legs straight; arms at sides

2. Lift both legs up to 90 degrees hip flexion; draw core into spine

Swimming on Knees
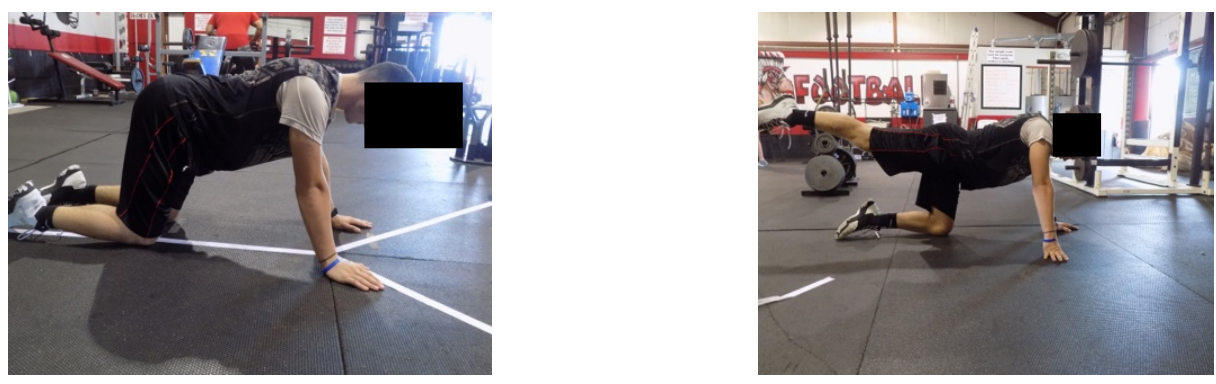

1. Begin in quadruped position (on hands and knees) with back straight

2. Raise leg to be parallel with the trunk

3. Lower to start position

4. Repeat with other leg 
Forward Lunge with Medicine Ball Twist
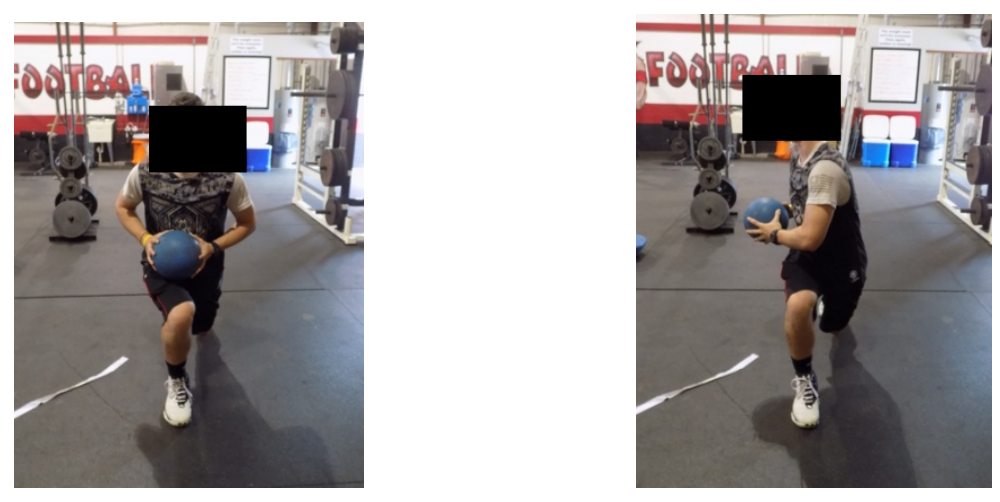

1. Begin standing with feet shoulder width apart holding the medicine ball

2. Step forward into lunge bringing back knee as far down to ground as possible; medicine ball held chest level

3. Rotate body right then left maintaining medicine ball at chest level

4. Return to beginning stance then repeat stepping out on opposite leg

Standing Reverse Wood Chop
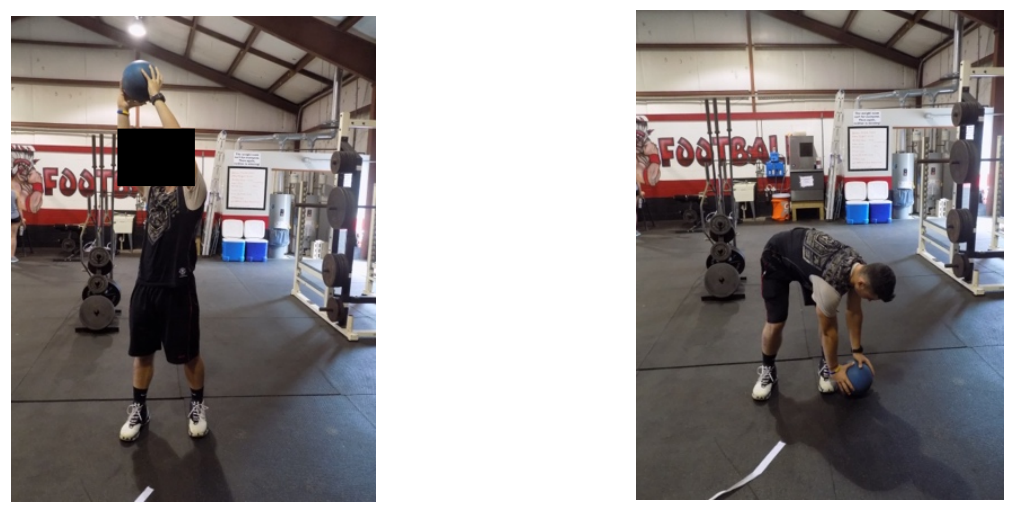

1. Begin standing with feet shoulder width apart holding the medicine ball

2. While holding the medicine ball, the ball moves overhead to the right then cut through the ground on the left of the body.

3. The exercise is then repeated upper left to lower right. 
Single Leg Squat while dribbling

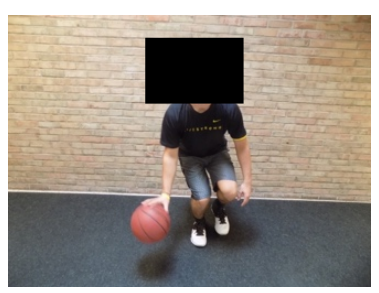

1. Begin with feet shoulder width apart.

2. Raise left leg off the ground.

3. Squat with the right leg keeping the knee over toes and keeping the back straight Bosu Squats while shooting basketball
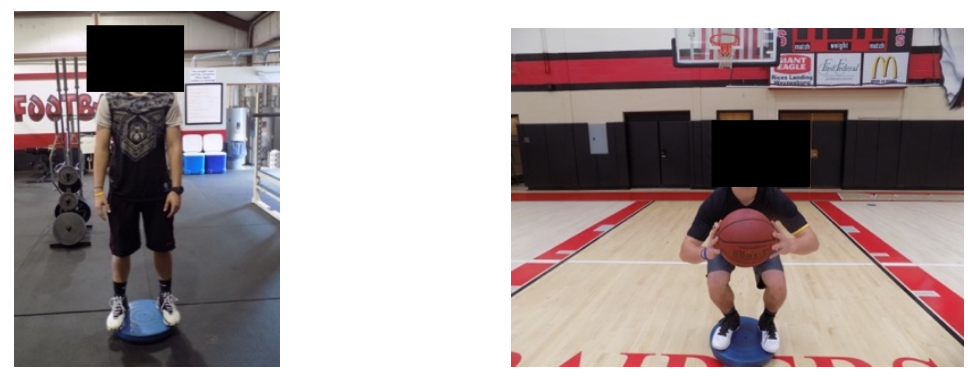

1. Begin with feet shoulder width apart with weight distributed equally on Bosu ball

2. Assume squat position with knee over toes and back straight

Push Ups

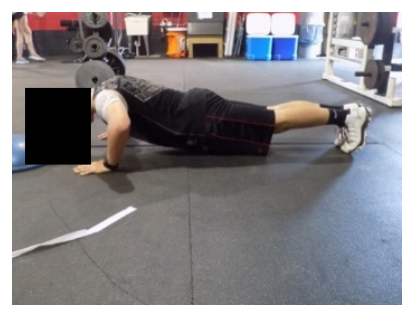

1. Begin in a plank position on your hands, shoulder width apart, and toes; Core drawn in; Back straight.

2. Lower down to ground until elbows reach 90 degree angle; Do not touch the ground; Maintain straight back and draw core in to spine 
Medicine Ball Throw
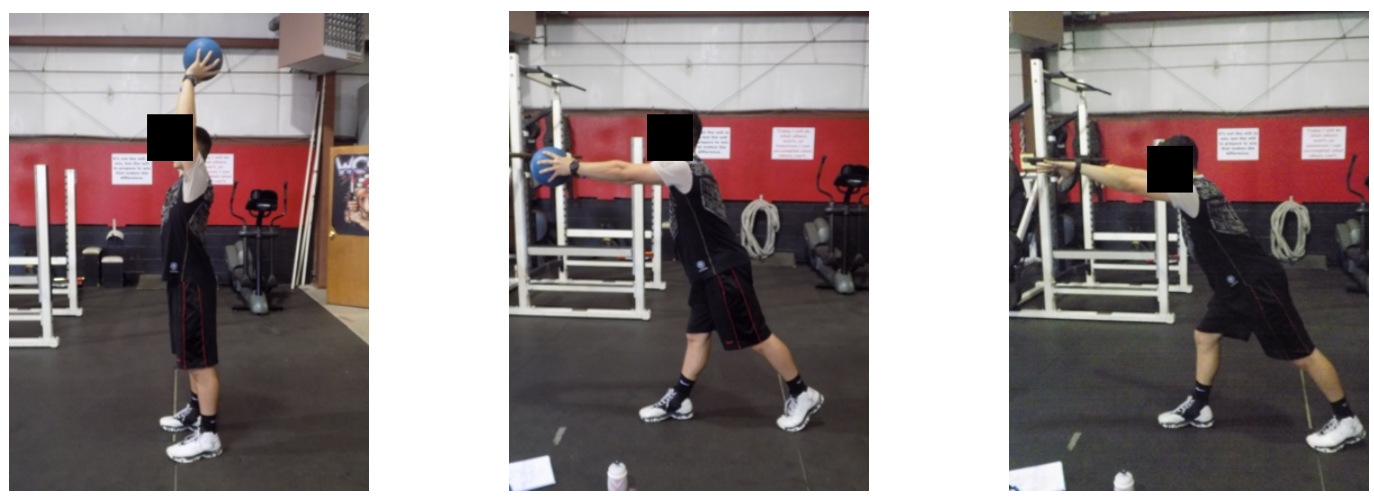

1. Begin standing feet shoulder width apart; Medicine ball held straight above head

2. Step with lead foot in order to lunge into the throw

3. Release the medicine ball

Dumbbell Forward Bend
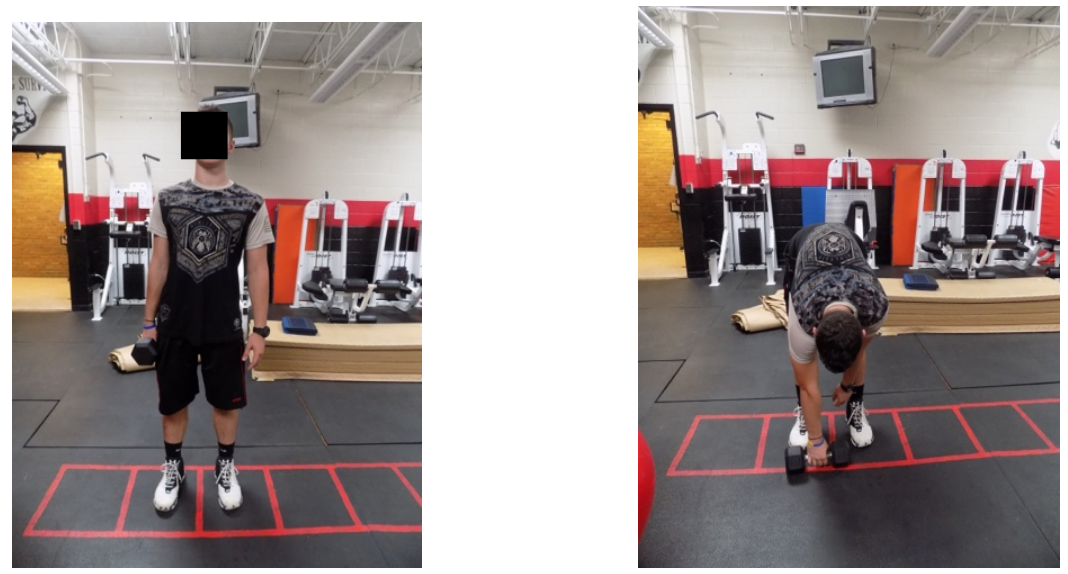

1. Begin standing feet shoulder width apart; Dumbbell in hand on one side

2. Bend over at waist while contracting core muscles

3. Repeat exercise with dumbbell in other hand 
Table C12. Lumbopelvic Control Intervention Phase 6 Exercises

Multidirectional Lunges w/ Med Ball Twist
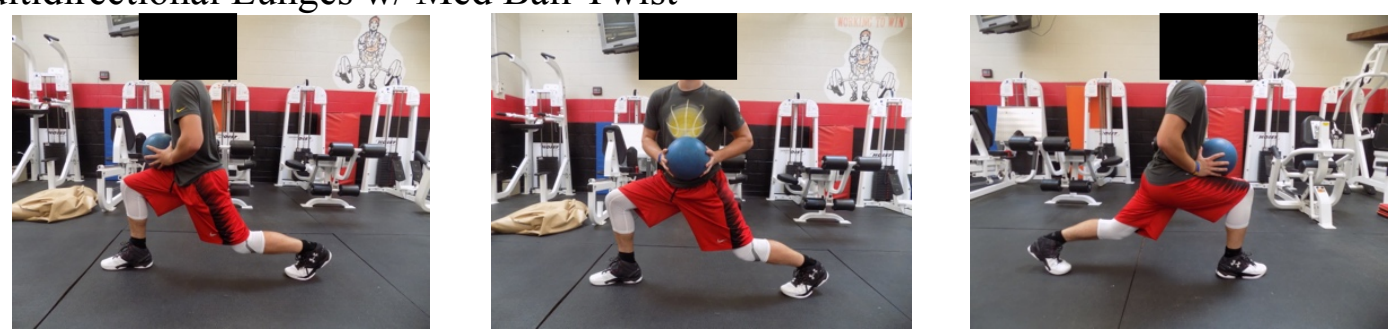

1. Begin standing with feet shoulder width apart holding the medicine ball

2. Step forward into lunge bringing back knee as far down to ground as possible; medicine ball held chest level

3. Rotate body right then left maintaining medicine ball at chest level

4. Return to beginning stance then repeat stepping out to the right then the left

5. Repeat exercise using other leg as lead leg

Uneven Single Leg Squat while dribbling
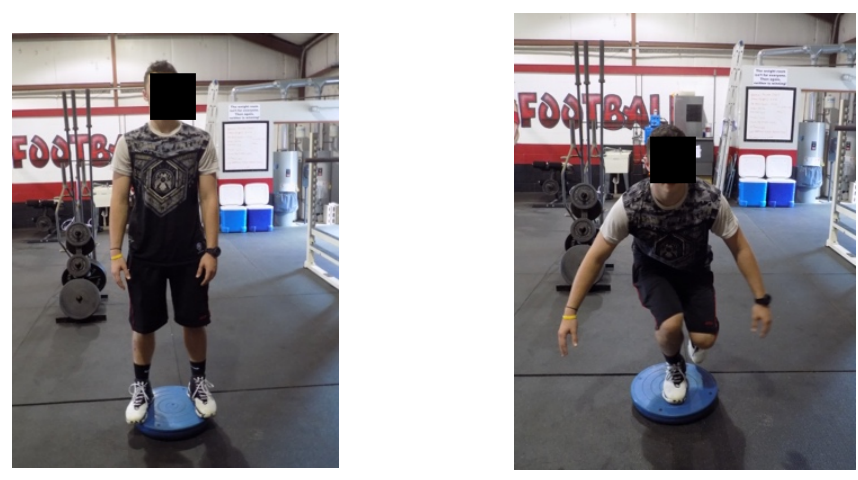

1. Begin with feet shoulder width apart on Bosu ball

2. Raise left leg off the ground.

3. Squat with the right leg keeping the knee over toes and keeping the back straight

4. Repeat exercise on other leg 
Bosu Squat with Medicine Ball Twist
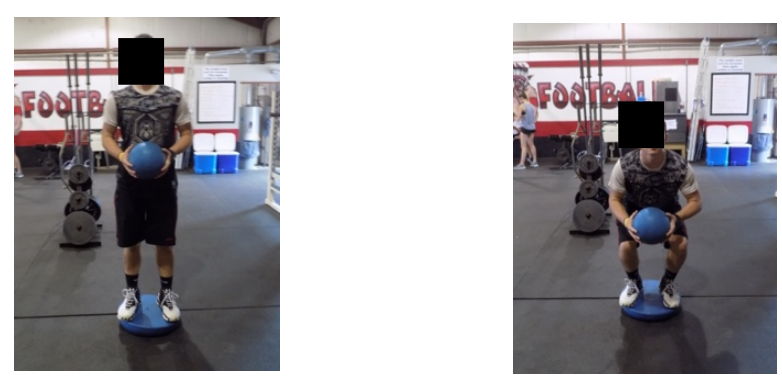

1. Begin with feet shoulder width apart with weight distributed equally on Bosu ball

2. Assume squat position with knee over toes and back straight

3. Rotate body right then left maintaining medicine ball at chest level

Dumbbell Side Bends
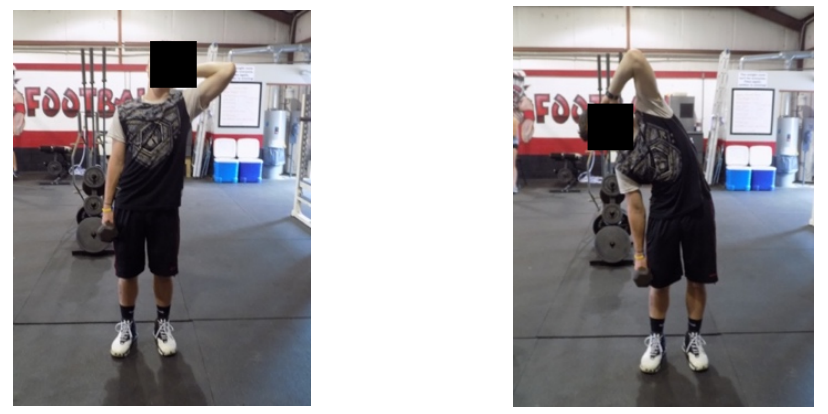

1. Begin standing feet shoulder width apart; Dumbbell in hand on one side; Other hand on head

2. Laterally bend at waist while contracting core muscles

3. Repeat exercise with dumbbell in other hand

Unstable Push Ups
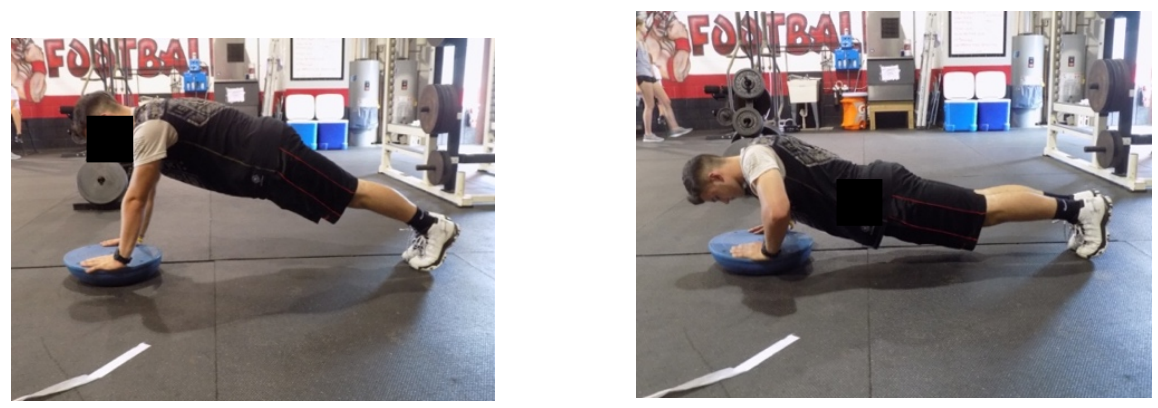
1. Begin in a plank position with your hands on the Bosu ball, shoulder width apart, and toes; Core drawn in; Back straight

2. Lower down to ground until elbows reach 90 degree angle; Do not touch the ground; Maintain straight back and draw core in to spine

Medicine Ball Slam
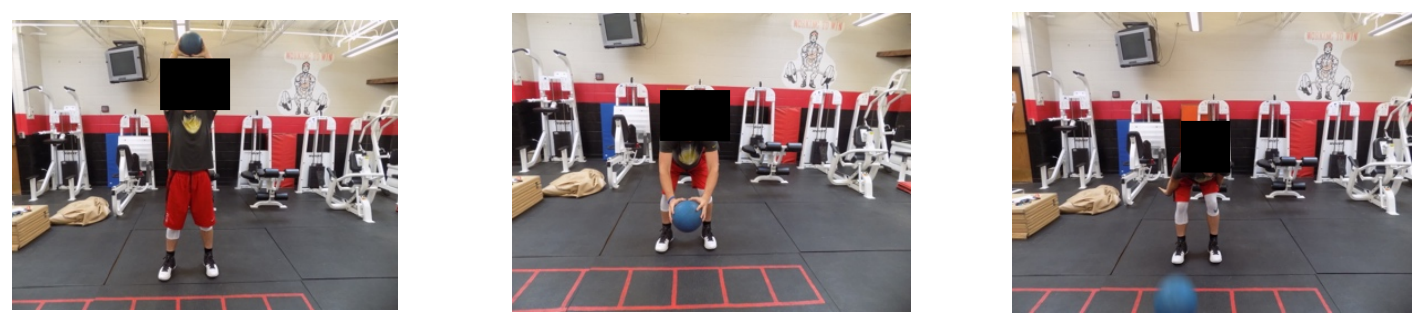

1. Begin standing feet shoulder width apart; Medicine ball held straight above head; Core drawn in to spine

2. Throw medicine ball hard at the ground using entire body with slight bend in knees

Side Step Lunge with Wood Chop
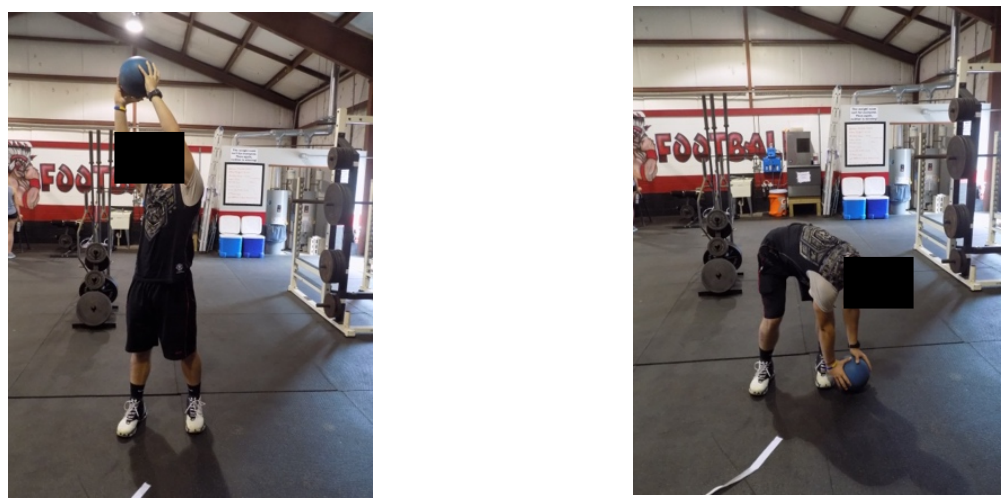

1. Begin standing with feet shoulder width apart holding the medicine ball

2. While holding the medicine ball, the ball moved overhead to the right then cut through the ground on the left of the body.

3. The exercise is then repeated upper left to lower right. 


\section{APPENDIX D}

\section{ADDITIONAL RESULTS}

Table D1. Descriptive Statistics on Means and Standard Deviations for Dependent Variables

\begin{tabular}{ccc}
\hline Dependent Variables & Pre-Test & Post Test \\
\hline SLRTL (degrees) & $13.17 \pm 14.00$ & $1.96 \pm 3.03$ \\
SLRTR (degrees) & $13.31 \pm 19.79$ & $3.21 \pm 2.47$ \\
AFT (seconds) & $71.64 \pm 28.41$ & $119.39 \pm 115.05$ \\
SEBT AL (\% of leg length) & $71.47 \pm 7.70$ & $65.46 \pm 28.55$ \\
SEBT PML (\% of leg length) & $92.39 \pm 11.23$ & $86.30 \pm 37.76$ \\
SEBT PLL (\% of leg length) & $104.18 \pm 13.20$ & $97.87 \pm 42.97$ \\
SEBT AR (\% of leg length) & $74.79 \pm 7.19$ & $67.45 \pm 29.17$ \\
SEBT PMR (\% of leg length) & $93.71 \pm 10.44$ & $86.04 \pm 37.32$ \\
SEBT PLR (\% of leg length) & $104.38 \pm 9.88$ & $92.98 \pm 40.22$ \\
\hline
\end{tabular}

Key: SLRTL = Single Leg Raise Test Left Leg; SLRTR= Single Leg Raise Test Right Leg; AFT= Abdominal Fatigue Test; SEBT AL= Star Excursion Balance Test Anterior Left Leg; SEBT PML= Star Excursion Balance Test Posteromedial Left Leg; SEBT PLL= Star Excursion Balance Test Posterolateral Left Leg; SEBT AR= Star Excursion Balance Test Anterior Right Leg; SEBT PMR= Star Excursion Balance Test Posteromedial Right Leg; SEBT PLR= Star Excursion Balance Test Posterolateral Right Leg

Table D2. Repeated Measures ANOVA Results

\begin{tabular}{cccccc}
\hline $\begin{array}{c}\text { Dependent } \\
\text { Variables }\end{array}$ & df & F & P & ES & $\mathbf{9 5 \%}$ CI \\
\hline SLRTL & 1 & 7.56 & 0.017 & -0.80 & -1.57 to \\
SLRTR & 1 & 3.24 & 0.095 & -0.51 & -1.26 to \\
& & & & 0.24 \\
AFT & 1 & 3.42 & 0.087 & 1.68 & 0.82 to \\
SEBT AL & 1 & 0.59 & 0.458 & -0.78 & -1.55 to \\
SEBT PML & 1 & 0.46 & 0.508 & -0.54 & -1.30 to \\
& & & & & 0.21 \\
SEBT PLL & 1 & 0.37 & 0.556 & -0.48 & -1.23 to \\
SEBT AR & 1 & 0.75 & 0.401 & -1.02 & -1.81 to \\
& & & & -0.23 \\
SEBT PMR & 1 & 0.56 & 0.468 & -0.73 & -1.50 to \\
SEBT PLR & 1 & 1.03 & 0.328 & -1.15 & -1.95 to - \\
& & & & & 0.35 \\
\hline
\end{tabular}

Key: SLRTL= Single Leg Raise Test Left Leg; SLRTR= Single Leg Raise Test Right Leg; AFT= Abdominal Fatigue Test; SEBT AL= Star Excursion Balance Test Anterior Left Leg; SEBT PML= Star Excursion Balance Test Posteromedial Left Leg; SEBT PLL= Star Excursion Balance Test Posterolateral Left Leg; SEBT AR= Star Excursion Balance Test Anterior Right Leg; SEBT PMR= Star Excursion Balance Test Posteromedial Right Leg; SEBT PLR= Star Excursion Balance Test Posterolateral Right Leg 


\section{APPENDIX E}

\section{RECOMMENDATIONS FOR FUTURE RESEARCH}

1. Include use of the female population

2. Use other sport's teams such as soccer, football, and volleyball

3. Conduct study with the recreational, collegiate, and youth athlete populations

4. Conduct study toward beginning of the season to promote motivation.

5. Conduct study solely during the off-season to see if in-season participation affects results

6. Substitute the Star Excursion Balance Test with the Jump Drop test for testing

7. Skip the first two phases with easy activation exercises and start the intervention with phase 3 and 4

8. Extend the intervention program time frame to eight or twelve weeks.

9. Incorporate more balance exercises to improve dynamic balance such as single leg stabilization progressions 


\section{ADDITIONAL REFERENCES}

41. McGill SM, Grenier S, Kavcic N, Cholewicki J. Coordination of muscle activity to assure stability of the lumbar spine. J Electromyogr Kinesiol. 2003;13(4):353-359.

42. Kahle NL, Gribble PA. Core stability training in dynamic balance testing among young, healthy adults. Athl Train Sport Health Care. 2009;1(2):65-73.

43. S, Voorst L, Van C. Everyone's playing basketball. Teach Child Math. 2001;7(9):518519.

44. Brenner JS. Sports specialization and intensive training in young athletes. Ped. 2016;138(3)

45. Sharrock C, Cropper J, Mostad J, Johnson M, Malone T. A pilot study of core stability and athletic performance: is there a relationship?. Int J Sports Phys Ther. 2011;6(2):6374.

46. Struzik A, Zawadzki J, Pietraszewski B. Balance disorders caused by running and jumping occurring in young basketball players. Acta Bioeng Biomech. 2015;17(2):103109.

47. Riemann BL, Lephart SM. The sensorimotor system, part I: the physiologic basis of functional joint stability. J Athl Train. 2002;37(1):71-79.

48. Arokoski JP, Valta T, Airaksinen O, Kankaanpää M. Back and abdominal muscle function during stabilization exercises. Arch Phys Med Rehabil. 2001;82(8):1089-1098.

49. McGill S. Low back disorders: evidence-based prevention and rehabilitation. Champaign, 111: Human Kinetics, 2002,

50. McGill SM, Karpowicz A. Exercises for spine stabilization: motion/motor patterns, stability progressions, and clinical technique. Arch Phys Med Rehabil. 2009;90(1):118126.

51. Drysdale CL, Earl JE, Hertel J. Surface electromyographic activity of the abdominal muscles during pelvic-tilt and abdominal-hollowing exercises. J Athl Train. 2004;39(1):32-36.

52. Konin JG, Peterson CL. Strengthening the core from the inside out. Athl Ther Today. 2003;8(4):41-43.

53. Cholewicki J, Panjabi MM, Khachatryan A. Stabilizing function of trunk flexor-extensor muscles around a neutral spine posture. Spine. 1997;22(19):2207-2212. 
54. King MA. Core stability: creating a foundation for functional rehabilitation. Athl Ther Today. 2000;5(2):6-13.

55. Hodges PW, Richardson CA: Contraction of the abdominal muscles associated with movement of the lower limb. Phys Ther 1997;77:132-142.

56. Weir A, Darby J, Inklaar H, Koes B, Bakker E, Tol JL. Core stability: inter- and intraobserver reliability of 6 clinical tests. Clin J Sport Med. 2010;20(1):34-38.

57. Lephart SM, Pincivero DM, Rozzi SL. Proprioception of the ankle and knee. Sports Med. 1998;25(3):149-155.

58. Winter DA, Patla AE, Frank JS. Assessment of balance control in humans. Med Prog Technol. 1990;16(1-2):31-51.

59. Tse MA, Mcmanus AM, Masters RS. Development and validation of a core endurance intervention program: implications for performance in college-age rowers. J Strength Cond Res. 2005;19(3):547-552.

60. Dale RB, Samson KM, Sandrey MA, Hetrick A. A core stabilization training program for tennis athletes. Athl Ther Today. 2007;12(3):41-46.

61. Cohen SB, Whiting WC, Mclaine AJ. Implementation of balance training in a gymnast's conditioning program. Strength Condit J. 2002;24(2):60-67. 